\title{
Charged polymers in the attractive regime: a first-order transition from Brownian scaling to four-point localization
}

\author{
Yueyun $\mathrm{Hu}$ \\ Davar Khoshnevisan* \\ Marc Wouts \\ Université Paris 13 \\ University of Utah \\ Université Paris 13
}

November 2, 2010

\begin{abstract}
We study a quenched charged-polymer model, introduced by Garel and Orland in 1988, that reproduces the folding/unfolding transition of biopolymers. We prove that, below the critical inverse temperature, the polymer is delocalized in the sense that: (1) The rescaled trajectory of the polymer converges to the Brownian path; and (2) The partition function remains bounded.

At the critical inverse temperature, we show that the maximum time spent at points jumps discontinuously from 0 to a positive fraction of the number of monomers, in the limit as the number of monomers tends to infinity.

Finally, when the critical inverse temperature is large, we prove that the polymer collapses in the sense that a large fraction of its monomers live on four adjacent positions, and its diameter grows only logarithmically with the number of the monomers.

Our methods also provide some insight into the annealed phase transition and at the transition due to a pulling force; both phase transitions are shown to be discontinuous.
\end{abstract}

${ }^{*}$ Research supported by NSF grants DMS-0706728 and DMS-1006903 
Keywords: Charged polymers, quenched measure, annealed measure, localization-delocalization transition, first-order phase transition.

AMS 2000 subject classification: Primary: 60K35; Secondary: 60K37.

\section{Introduction and main results}

\subsection{The charged polymer model}

We consider a polymer model introduced by Garel and Orland in [9] for modeling the trajectory of biological proteins made of hydrophobic monomers. Let $\left\{q_{i}\right\}_{i=0}^{\infty}$ be i.i.d. real variables and $\left\{S_{i}\right\}_{i=0}^{\infty}$ an independent simple random walk on $\mathbf{Z}^{d}$ with $S_{0}=0$. Both stochastic processes exist on a common probability space $(\Omega, \mathcal{F}, \mathrm{P})$.

Given a realization of $q$ and $S$, we consider

$$
\begin{aligned}
Q_{N}^{x} & :=\sum_{0 \leqslant i<N} q_{i} \mathbf{1}_{\left\{S_{i}=x\right\}}, \quad \text { and } \\
H_{N} & :=\sum_{x \in \mathbf{Z}^{d}}\left(Q_{N}^{x}\right)^{2} .
\end{aligned}
$$

We think of the $q_{i}$ 's as charges, $Q_{N}^{x}$ as the total charge at position $x \in \mathbf{Z}^{d}$, and $H_{N}$ as the energy of the polymer. In this way, we see that $Q_{N}^{x}$ and $H_{N}$ in fact define functions of the trajectory $S$ of the walk. Therefore, we might occasionally refer to them respectively as $Q_{N}^{x}(S)$ and $H_{N}(S)$, as well.

For all $\beta \in \mathbf{R}$ [inverse temperature] and $N \geqslant 1$ [the number of monomers] consider the quenched probability measure $\mathrm{P}_{N}^{\beta}$,

$$
\mathrm{P}_{N}^{\beta}(A):=\frac{1}{Z_{N}(\beta)} \mathrm{E}\left[\mathbf{1}_{A} \exp \left(\frac{\beta}{N} H_{N}\right) \mid q_{0}, q_{1}, \ldots, q_{N-1}\right],
$$

where $Z_{N}(\beta)$ [the partition function] is defined so that $\mathrm{P}_{N}^{\beta}$ is indeed a probability measure; that is,

$$
Z_{N}(\beta):=\mathrm{E}\left[\exp \left(\frac{\beta}{N} H_{N}\right) \mid q_{0}, q_{1}, \ldots, q_{N-1}\right]
$$


We can write the energy in the following equivalent form:

$$
H_{N}:=2 \hat{H}_{N}+\sum_{0 \leqslant i<N} q_{i}^{2}
$$

where

$$
\hat{H}_{N}:=\sum_{0 \leqslant i<j<N} \sum_{i} q_{j} \mathbf{1}_{\left\{S_{i}=S_{j}\right\}}
$$

Therefore, if we define the quenched measure $\widehat{\mathrm{P}}_{N}^{\beta}$ as we did $\mathrm{P}_{N}^{\beta}$ but with $\hat{H}_{N}$ in place of $H_{N}$, then $\widehat{\mathrm{P}}_{N}^{\beta}=\mathrm{P}_{N}^{\beta / 2}$. Thus, the analyses of $\mathrm{P}_{N}^{\beta}$ and $\widehat{\mathrm{P}}_{N}^{\beta}$ are the same, but one has to remember to halve/double the parameter $\beta$ in order to understand one in terms of the other.

In our model, like charges attract when $\beta>0$. This accounts for the hydrophobic properties of monomers immersed in water [9]. And the scaling $H_{N} / N$ was introduced also in [9] in order to compensate for the absence of hard-core repulsion. It will also follow from Lemma 2.5 below that this scaling makes the energy subadditive [or extensive]. The fact that charges interact only when they are at exactly the same position is said to account for the screening effect: When a polymer is immersed in water, its charges are surrounded by oppositely-charged free molecules of the solvent.

Garel and Orland $[9,10]$ introduced the charged-polymer model in order to better understand the transition, in biopolymers, from a swollen state to a folded state. In [10] the authors perform a mean-field analysis of a model with independent, Gaussian interactions between monomers pairs. And in [9] they introduce [a generalization of] $\hat{H}_{N}$ in order to model different possible attractive/repulsive forces between different monomers such as amino acids in proteins or the base-pairs in the RNA. ${ }^{1}$

When the reference random walk $\left\{S_{i}\right\}_{i=0}^{\infty}$ is replaced by a walk on a simplex with $d$ points, Garel and Orland [9] find a continuous phase transition from a folded to an unfolded state as the temperature increases. And, for a continuous version of the charged-polymer model, they find that a similar continuous phase transition holds at an explicit temperature. In another

\footnotetext{
${ }^{1}$ The energy in [9] corresponds to ours when their $M=1$.
} 
paper [10], however, Garel and Orland mention that the phase transition in biopolymers is expected to be discontinuous. Among other things, the results of our paper confirm their prediction in the present charged-polymer model.

The physics literature contains also the analyses of several seeminglysimilar models that are not equivalent to ours mainly because in those models like charges repel $[7,8,12,15]$.

In the last few years the mathematics of polymer measures has also grown considerably $[5,6,11,19]$. However, it appears that little is known about our model. We are aware only of Chapter 8 of [6] on the annealed measure in the repelling regime $\beta \leqslant 0$, and that result holds for a different scaling of the energy [for which the polymer is completely localized.]

We are aware also of some recent works on the energy $\hat{H}_{N}$ itself: In [3], limit theorems for $\hat{H}_{N}$ are established; it was shown in [4] that the distribution of $\hat{H}_{N}$ is comparable to the random walk in random scenery as $N$ tends to infinity, see also [13]; and large deviations for $\hat{H}_{N}$ were established in $[1,2]$.

Let us conclude this introduction with a brief outline of the paper: In the remainder of this section we present our main results on the model. Those results range from a characterization of the delocalized phase to a description of the discontinuous phase transition, and finally to large- $\beta$ asymptotics. We also emphasize some differences between the quenched and annealed measures, and describe the effect of a pulling force. Proofs of the various assertions are relegated to Section 2. Finally, we include some basic facts about the local times of the simple random walk in the appendix.

\subsection{The delocalized phase}

Unless it is stated to the contrary, we assume that $\mathrm{E} q_{0}=0$, $\operatorname{Var} q_{0}=1$, and that the charges are subgaussian; that is, $\kappa<\infty$, where

$$
\kappa:=\inf \left\{c \in(-\infty, \infty]: \operatorname{Ee}^{t q_{0}} \leqslant \mathrm{e}^{c t^{2} / 2} \quad \text { for all } \quad t \in \mathbf{R}\right\}
$$


We have $\kappa \geqslant 1$ as long as $q_{0}$ has a finite moment generating function near zero and $\mathrm{E} q_{0}=0$. And $\kappa=1$ both when the $q_{i}$ 's have the Rademacher distribution $\left[\mathrm{P}\left\{q_{0}= \pm 1\right\}=1 / 2\right]$ and when they have a standard normal distribution.

Now we introduce

$$
\mathscr{D}:=\left\{\beta \in \mathbf{R}: Z_{N}(\beta) \stackrel{\mathrm{P}}{\longrightarrow} \mathrm{e}^{\beta} \quad \text { as } N \rightarrow \infty\right\}
$$

where " $\stackrel{\mathrm{P}}{\longrightarrow}$ " denotes convergence in probability. As is customary, we call

$$
L_{N}^{x}:=\sum_{i=0}^{N-1} \mathbf{1}_{\left\{S_{i}=x\right\}}
$$

the local time of $\left\{S_{i}\right\}_{i=0}^{N-1}$ at $x$, and define

$$
L_{N}^{\star}:=\max _{x \in \mathbf{Z}^{d}} L_{N}^{x}
$$

to be maximum local time.

The next theorem tells us that the set $\mathscr{D}$ characterizes the region of $\beta$ for which the trajectory of the polymer is [asymptotically] indistinguishable from that of a random walk. In other words, the polymer is delocalized when $\beta \in \mathscr{D}$ and $N$ is large.

thm:D Theorem 1.1. If $\mathrm{E} q_{0}=0$, $\operatorname{Var} q_{0}=1$, and $\kappa<\infty$, then:

1. $\mathscr{D}$ is an interval that contains $(-\infty, 1 / \kappa)$.

2. $\beta \in \mathscr{D}$ if and only if for all $\varepsilon>0$,

$$
\mathrm{P}_{N}^{\beta}\left\{L_{N}^{\star} \leqslant \varepsilon N\right\} \stackrel{\mathrm{P}}{\longrightarrow} 1 \quad \text { as } N \rightarrow \infty .
$$

3. $\beta \in \mathscr{D}$ if and only if:

$$
\left\|\mathrm{P}_{N}^{\beta}-\mathrm{P}\left[\cdot \mid q_{0}, \ldots, q_{N-1}\right]\right\|_{\mathrm{TV}} \stackrel{\mathrm{P}}{\longrightarrow} 0 \quad \text { as } N \rightarrow \infty
$$

where $\|\mu-\nu\|_{\mathrm{TV}}:=\sup _{A}|\mu(A)-\nu(A)|$ is the total variation distance. 
In order to describe a consequence of Theorem 1.1, let $N \geqslant 1$ be an integer, and consider the stochastic process $\mathscr{S}_{N}$ defined by

$$
\mathscr{S}_{N}(t):=(N t-[N t])\left(\frac{S_{[N t]+1}-S_{[N t]}}{\sqrt{N}}\right)+\frac{S_{[N t]}}{\sqrt{N}} \quad(0 \leqslant t \leqslant 1) .
$$

$\mathscr{S}_{N}$ is defined uniquely as the piecewise-linear function that takes the values $S_{k} / \sqrt{N}$ at $t=k / N$ for all integers $k=0, \ldots, N$. Now we can mention the consequence of Theorem 1.1 .

cor: BM Corollary 1.2. If $\mathrm{E} q_{0}=0$, Var $q_{0}=1$, and $\kappa<\infty$, then for all $\beta \in \mathscr{D}$ and $\Phi: C([0,1]) \rightarrow \mathbf{R}$ bounded and continuous,

$$
\mathrm{E}_{N}^{\beta}\left[\Phi\left(\mathscr{S}_{N}\right)\right] \underset{N \rightarrow \infty}{\stackrel{\mathrm{P}}{\longrightarrow}} \mathrm{E}[\Phi(\mathscr{B})]
$$

where $\mathscr{B}$ denotes d-dimensional Brownian motion.

Remark 1.3. Even though $\beta \in \mathscr{D}$ if and only if $\mathrm{P}_{N}^{\beta}\left\{L_{N}^{\star}<\varepsilon N\right\} \rightarrow 1$ in probability, one can say more about the rate of this convergence when $\beta$ in the interior of $\mathscr{D}$. Indeed, suppose $\beta$ lies in the interior of $\mathscr{D}$. It follows from part 1 of Theorem 1.1 that $q \beta \in \mathscr{D}$ for some $q>1$. Let $p$ denote the conjugate to $q$; that is, $p^{-1}+q^{-1}=1$. Then Hölder's inequality implies that

$$
\mathrm{P}_{N}^{\beta}\left\{L_{N}^{\star} \geqslant \varepsilon N\right\} \leqslant\left[\mathrm{P}\left\{L_{N}^{\star} \geqslant \varepsilon N\right\}\right]^{1 / p} \cdot \frac{\left[Z_{N}(q \beta)\right]^{1 / q}}{Z_{N}(\beta)} .
$$

The fraction of the $Z_{N}$ 's goes to one in probability since both $\beta$ and $q \beta$ are in $\mathscr{D}$. Therefore, it follows from Lemma A.2 below that $\mathrm{P}_{N}^{\beta}\left\{L_{N}^{\star}<\varepsilon N\right\} \rightarrow 1$, in probability, exponentially fast, as long as $\beta$ lies in the interior of $\mathscr{D}$.

\subsection{A first-order phase transition}

We show, in Lemma 2.5 below, that the normalized energy $H_{N} / N$ is subadditive. And it will follow from that fact that the free energy $\digamma$ exists when the second moment of the charge distribution is finite. More precisely, we have the following. 
prop: $\mathrm{F}$ Proposition 1.4. If $\mathrm{E}\left(q_{0}^{2}\right)<\infty$, then for all $\beta \in \mathbf{R}$,

$$
\digamma(\beta):=\lim _{N \rightarrow \infty} \frac{1}{N} \ln Z_{N}(\beta)
$$

exists a.s. and in $L^{1}(\mathrm{P})$, and $\digamma(\beta)$ is nonrandom. The function $\mathbf{R} \ni \beta \rightarrow$ $\digamma(\beta)$ is nonnegative, nondecreasing, and convex with $\digamma(0)=0$.

Define the critical inverse temperature,

$$
\beta_{c}:=\sup \mathscr{D}
$$

Clearly, $\digamma(\beta)=0$ whenever $\beta \leqslant \beta_{c}$. We now wish to know whether or not the converse is true.

Our next theorem shows that a first-order phase transition occurs at $\beta_{c}$, and that the maximal fraction $L_{N}^{\star} / N$ of monomers on a single site jumps discontinuously from 0 to a quantity that is at least $1 /\left(2 \kappa \beta_{c}\right)>0$. It might help to recall that convex functions have right derivatives everywhere.

thm: fo Theorem 1.5. If $\mathrm{E} q_{0}=0$, Var $q_{0}=1$, and $\kappa<\infty$, then $\digamma\left(\beta_{c}\right)=0$, whereas $\digamma(\beta)>0$ for all $\beta>\beta_{c}$. Moreover, there is a first-order phase transition at $\beta_{c} ;$ i.e.,

$$
\lim _{\beta \downarrow \beta_{c}} \frac{\digamma(\beta)}{\beta-\beta_{c}} \in(0, \infty) .
$$

Furthermore, if $\beta>\beta_{c}$, then for all $\varepsilon>0$,

$$
\mathrm{P}_{N}^{\beta}\left\{\frac{L_{N}^{\star}}{N} \geqslant \frac{1-\varepsilon}{\beta} \max \left(\digamma(\beta), \frac{1}{2 \kappa}\right)\right\} \stackrel{\mathrm{P}}{\longrightarrow} 1 \quad \text { as } N \rightarrow \infty .
$$

\subsection{The folded phase}

When the inverse temperature $\beta$ is large, the polymer measure concentrates on the configurations with high energy. In dimensions $d \geqslant 2$ we will compute the [quenched] maximum of $H_{N}$. It turns out that that maximum is realized when the walk is concentrated on four points that define a square.

Recall that $a^{+}:=a \vee 0$ and $a^{-}:=(-a)^{+}$for all $a \in \mathbf{R}$. When $Z$ is a random variable and $\varepsilon \in\{-,+\}$ we always write $\mathrm{E} Z^{\varepsilon}$ as shorthand for 
$\mathrm{E}\left(Z^{\varepsilon}\right)\left[\right.$ and never for $\left.(\mathrm{E} Z)^{\varepsilon}\right]$.

prop:maxH:bc Proposition 1.6. If $d \geqslant 2$, then for all $\beta \in \mathbf{R}$,

$$
\liminf _{N \rightarrow \infty} \frac{1}{N} \ln Z_{N}(\beta) \geqslant\left[\frac{\left(\mathrm{E} q_{0}^{+}\right)^{2}+\left(\mathrm{E} q_{0}^{-}\right)^{2}}{2}\right] \beta-\ln (2 d) \quad \text { a.s. }
$$

Consequently, under the assumptions of Theorem 1.1 [that $\mathrm{E} q_{0}=0$, Var $q_{0}=$ 1 , and $\kappa<\infty]$, the critical inverse temperature satisfies

$$
\beta_{c} \leqslant \frac{2 \ln (2 d)}{\left(\mathrm{E} q_{0}^{+}\right)^{2}+\left(\mathrm{E} q_{0}^{-}\right)^{2}}
$$

We emphasize that, in the case that $\mathrm{E}\left|q_{0}\right|=\infty$, the preceding proposition tells us that $\digamma(\beta)=\infty$ a.s. for all $\beta>0$. That proposition also tells us that $\beta_{c} \leqslant 4 \ln (2 d)$ when $q_{0}$ has the Rademacher distribution [i.e., $q_{0}= \pm 1$ with probability $1 / 2$ each] and $\beta_{c} \leqslant 2 \pi \ln (2 d)$ when $q_{0}$ has a standard normal distribution.

In order to prepare for our next results we first define the following quantities:

$$
\begin{aligned}
\gamma & :=\min _{\varepsilon \in\{-,+\}}\left(\mathrm{E} q_{0}^{\varepsilon}\right)^{2} ; \\
\lambda & :=\min _{\varepsilon, \varepsilon^{\prime} \in\{-,+\}} \mathrm{E}\left[\min \left(\left(\mathrm{E} q_{0}^{\varepsilon}\right) q_{0}^{\varepsilon},\left(\mathrm{E} q_{1}^{\varepsilon^{\prime}}\right) q_{1}^{\varepsilon^{\prime}}\right)\right] ; \quad \text { and } \\
\beta_{\alpha} & :=\ln (2 d) \cdot\left[\frac{8}{(1-\alpha) \gamma} \vee \frac{4}{\lambda}\right] \quad(0<\alpha<1) .
\end{aligned}
$$

We are interested mainly in $\beta_{\alpha}\left[\beta_{\alpha}\right.$ should not be confused with the critical inverse temperature $\beta_{c}$.]

It is possible to check that when $q_{0}$ has a symmetric distribution [i.e., $q_{0}$ 
and $-q_{0}$ have the same law],

$$
\begin{aligned}
\gamma & =\left(\mathrm{E} q_{0}^{+}\right)^{2}=\left(\int_{0}^{\infty} \mathrm{P}\left\{q_{0}>z\right\} \mathrm{d} z\right)^{2}, \\
\lambda & =\sqrt{\gamma} \cdot \mathrm{E}\left(q_{0}^{+} \wedge q_{1}^{+}\right)=\sqrt{\gamma} \cdot \int_{0}^{\infty}\left(\mathrm{P}\left\{q_{0}>z\right\}\right)^{2} \mathrm{~d} z \\
\beta_{\alpha} & =\frac{4 \ln (2 d)}{\sqrt{\gamma}} \cdot\left[\frac{2}{(1-\alpha) \sqrt{\gamma}} \vee \frac{1}{\mathrm{E}\left(q_{0}^{+} \wedge q_{1}^{+}\right)}\right] .
\end{aligned}
$$

Thus, for example, $\gamma=1 / 4, \lambda=1 / 8$, and $\beta_{\alpha}=32 \ln (2 d) /(1-\alpha)$ when $q_{0}$ has the Rademacher distribution $\left[q_{0}= \pm 1\right.$ with probability $1 / 2$ each].

In addition to the preceding constants, we will need some notation: We say that " $U$ is a unit square" if we can write $U=\left\{x_{1}, \ldots, x_{4}\right\}$ as a collection of four points that satisfy $\left\|x_{2}-x_{1}\right\|=\left\|x_{3}-x_{2}\right\|=\left\|x_{4}-x_{3}\right\|=\left\|x_{1}-x_{4}\right\|=1$.

Also, for $0<\alpha<1$ we define the event $\mathcal{S}_{\alpha}$,

$$
\mathcal{S}_{\alpha}:=\left\{\begin{array}{c}
\text { There exists a unique unit square } U \subset \mathbf{Z}^{d} \text { such that } \\
\sum_{1 \leqslant i<N: S_{i} \notin U}\left|q_{i}\right| \leqslant \frac{1-\alpha}{2} \sum_{1 \leqslant i<N}\left|q_{i}\right|
\end{array}\right\} .
$$

In other words, the event $\mathcal{S}_{\alpha}$ is realized exactly when there exists a unique unit square $U$ such that the sum of the absolute charges not on $U$ is at most $(1-\alpha) / 2$ times the total absolute charge of the polymer.

thm:square Theorem 1.7 (The four points). Assume $d \geqslant 2$. Then for all $\delta>0$, there is $c_{\delta} \in(0, \infty)$ such that for every $N \geqslant 1$ and $\beta \in \mathbf{R}$,

$$
\mathrm{P}\left\{\mathrm{P}_{N}^{\beta}\left(\mathcal{S}_{\alpha}\right) \geqslant 1-\exp \left(N \ln (2 d)\left[1-\frac{\beta}{(1+\delta) \beta_{\alpha}}\right]\right)\right\} \geqslant 1-\exp \left(-c_{\delta} N\right)
$$

Our result is limited to $d \geqslant 2$, since this is the minimal dimension in which we can consider a square. But other results are also sometimes possible. For example, if $S$ is replaced by the lazy random walk, then one can adapt the present methods to prove the existence of two adjacent points that bear most of the available charge provided that $\beta$ is large enough. And the latter assertion is valid for every $d \geqslant 1$. 
In the usual scaling $\beta H_{N} / N$, Theorem 1.7 shows that the polymer is localized for any $\beta>\beta_{\alpha}$. But the latter theorem yields a pointwise estimate in $\beta$. It is instructive to also consider the scaling in which $\beta=b N$ for some $b>0$. That is the case in which $\beta$ is proportional to $N$ instead of being a constant. In that case, $\mathcal{S}_{\alpha}$ continues to be a typical event when $\alpha=1-8 \ln (2 d) /((1+2 \delta) b \gamma N)$. In other words, for every $b>0$, all but a bounded amount of the absolute charges live on four points.

Given a nonempty subset $A \subset \mathbf{Z}^{d}$, define

$$
\operatorname{Diam} A:=\sup _{x, y \in A}\|x-y\|_{1}:=\sup _{x, y \in A} \sum_{i=1}^{d}\left|x_{i}-y_{i}\right|
$$

this defines the diameter of $A$. Our next result describes the behavior of the polymer for large values of $\beta$.

thm:range Theorem 1.8 (Logarithmic diameter). For all $\beta \in \mathbf{R}$ and $K>0$ there exist $0 \leqslant c \leqslant C \leqslant \infty$ such that

$$
\mathrm{E}\left[\mathrm{P}_{N}^{\beta}\left\{c \leqslant \frac{\operatorname{Diam}\left\{S_{i}: 0 \leqslant i<N\right\}}{\ln N} \leqslant C\right\}\right] \geqslant 1-N^{-K},
$$

for all sufficiently large integers $N \geqslant 1$. Moreover:

1. If $d \geqslant 1$ and $\mathrm{E}\left|q_{0}\right|<\infty$, then $c>0$.

2. If $d \geqslant 2$ and

$$
\beta>\min _{\alpha \in(0,1)}\left[\beta_{\alpha} \vee \frac{\ln (2 d)}{\alpha \sqrt{\gamma} \mathrm{E}\left|q_{0}\right|}\right]
$$

then $C<\infty$.

Therefore, the polymer is "compact" for large values of $\beta$ in the sense that its diameter grows only logarithmically with the number of monomers. Remark 1.9. Note, for example, that when the charges have the Rademacher distribution [i.e., $q_{0}= \pm 1$ with probability $1 / 2$ each], condition (1.29) is stating that $\beta>34 \ln (2 d)$. 
Remark 1.10. Our proof applies equally well to the case that $\beta$ scales with $N$ (see Theorem 2.15). And the endresult is that, in order to have a "bounded diameter," it suffices that $\beta=b \ln N$ for some $b>0$.

Although the range of the polymer diverges with $N$ (Theorem 1.8), one can show that the expectation of $\left\|S_{N}\right\|$ remains bounded for all $\beta$ sufficiently large. We describe this phenomenon next.

Given $\alpha \in(0,1)$ consider the random variable

$$
R_{\alpha}^{N}:= \begin{cases}\inf \left\{0 \leqslant i<N: S_{i} \in U\right\} & \text { on } \mathcal{S}_{\alpha}, \\ N & \text { on } \mathcal{S}_{\alpha}^{c}\end{cases}
$$

where $U$ is the unique random square that concentrates most of the charges, given $\mathcal{S}_{\alpha}$. The quantity $R_{\alpha}^{N}$ is therefore the index of the first monomer that belongs to the unit square $U$ on $\mathcal{S}_{\alpha}$. And one can use $R_{\alpha}^{N}$ in order to obtain a bound on the distance from $U$ to the origin. The distributional symmetry of the polymer shows that the last monomer on $U$ has the same distribution as $N-1-R_{\alpha}^{N}$. Therefore, for any $0<\alpha<1$,

$$
\mathrm{EE}_{N}^{\beta}\left|S_{N}\right| \leqslant \sqrt{2}+2 \mathrm{EE}_{N}^{\beta}\left(R_{\alpha}^{N}\right)
$$

We will prove later on that the distribution of $R_{\alpha}^{N}$ has an exponential tail. Our final result is:

thm:ER Theorem 1.11 (Compactness). Suppose $d \geqslant 2, \alpha \in(0,1), \beta>\beta_{\alpha}$, and

$$
\rho:=2 d \mathrm{E}\left(\mathrm{e}^{-\beta \alpha \sqrt{\gamma}\left|q_{0}\right|}\right)<1
$$

Then,

$$
\limsup _{N \rightarrow \infty} \mathrm{EE}_{N}^{\beta}\left(R_{\alpha}^{N}\right) \leqslant \frac{\rho}{(1-\rho)^{2}} .
$$

Condition (1.32) is frequently easy to check. For example, when $q_{0}$ has the Rademacher distribution [i.e., $\mathrm{P}\left\{q_{0}= \pm 1\right\}=1 / 2$ ], $\rho=2 d \exp (-\beta \alpha / 2)$, and (1.32) holds if and only if $\beta>2 \ln (2 d) / \alpha$. Since $\beta_{\alpha}=32 \ln (2 d) /(1-\alpha)$, 
we find that - in the case of Rademacher-distributed charges - we have

$$
\beta>34 \ln (2 d) \quad \Longrightarrow \quad \sup _{N \geqslant 1} \mathrm{EE}_{N}^{\beta}\left(R_{1 / 17}^{N}\right) \leqslant \frac{\rho}{(1-\rho)^{2}}<\infty
$$

\subsection{On the annealed measure}

Our proofs can be easily adapted to describe the behavior of the annealed measure, defined by

$$
\widetilde{\mathrm{P}}_{N}^{\beta}(A):=\frac{1}{\mathrm{E} Z_{N}(\beta)} \mathrm{E}\left[\mathbf{1}_{A} \exp \left(\frac{\beta}{N} H_{N}\right)\right]
$$

when $\mathrm{E} Z_{N}(\beta)<\infty$. (The latter condition holds, for example, when $\beta<1 / \kappa$ and $N$ is sufficiently large). The annealed free energy is

$$
\widetilde{\digamma}(\beta):=\lim _{N \rightarrow \infty} \frac{1}{N} \ln \mathrm{E} Z_{N}(\beta) .
$$

We can define the region of delocalization for the annealed measure and the annealed critical point respectively as follows:

$$
\begin{aligned}
& \widetilde{\mathscr{D}}:=\left\{\beta \in \mathbf{R}: \lim _{N \rightarrow \infty} \mathrm{E} Z_{N}(\beta)=\mathrm{e}^{\beta}\right\} ; \\
& \tilde{\beta}_{c}:=\sup \widetilde{\mathscr{D}} .
\end{aligned}
$$

Our results for the annealed measure are similar in flavor to those for the quenched measure:

1. The set $\widetilde{\mathscr{D}}$ is an interval that contains $(-\infty, 1 / \kappa)$; it coincides with the localized phase in the sense that $\left\|\widetilde{\mathrm{P}}_{N}^{\beta}-\mathrm{P}\right\|_{\mathrm{TV}}$ converges to 0 as $N \rightarrow \infty$ if and only if $\beta \in \widetilde{\mathscr{D}}$.

2. Theorem 1.5 continues to remain valid after we replace $\beta_{c}$ by $\tilde{\beta}_{c}$ and $\digamma$ by $\widetilde{\digamma}$, and also add the restriction - to the set of $\beta$ 's - that $\operatorname{E} Z_{N}(\beta)$ is finite for all large $N$.

3. The proof of Lemma 2.4 shows that $\widetilde{\mathscr{D}} \subset \mathscr{D}$, therefore $\tilde{\beta}_{c} \leqslant \beta_{c}$; but we believe that this inequality is not sharp in general. 
It is sometimes possible to compute $\tilde{\beta}_{c}$; the following highlights an example.

Proposition 1.12. If $q_{0}$ has a standard normal distribution, then $\mathrm{E} Z_{N}(1)=$ $\infty$ for all $N \geqslant 1$. Consequently, $\tilde{\beta}_{c}=1$.

We can adapt many of our localization results to the annealed case provided that $\mathrm{E} Z_{N}(\beta)$ is finite and $\beta$ is large [consider for instance charges that are bounded random variables]. In those cases, as $\beta \rightarrow \infty$ the trajectory concentrates on two points, while the charges at a given parity tend to have a constant sign and an absolute value close to the essential supremum $\left\|q_{0}\right\|_{L^{\infty}(\mathrm{P})}$ of the charge distribution.

\subsection{The influence of a pulling force}

Our proofs will rely only very little on the assumption that $\left\{S_{i}\right\}_{i=0}^{\infty}$ is a simple symmetric random walk. To illustrate, let us say a few words about the case where $\left\{S_{i}\right\}_{i=0}^{\infty}$ has a bias that corresponds to the action of a pulling force.

For every $\lambda \in \mathbf{R}^{d}$ let us define a probability measure $\mathrm{P}_{\lambda}$ by the following prescription of its Radon-Nikodým derivative with respect to P: For every integer $k \geqslant 1$,

$$
\frac{\mathrm{dP}_{\lambda}}{\mathrm{dP}}:=\frac{\exp \left(\lambda \cdot S_{k}\right)}{\operatorname{E} \exp \left(\lambda \cdot S_{k}\right)} \quad \text { on } \mathcal{F}_{k}
$$

where $\mathcal{F}_{k}$ denotes the sigma-algebra generated by all of the charges $\left\{q_{i}\right\}_{i=0}^{\infty}$ as well as the $k$ initial values $\left\{S_{i}\right\}_{i=0}^{k}$ of the random walk.

Under the measure $\mathrm{P}_{\lambda}$ the distribution of the charges $q$ remains the same as that under $\mathrm{P}$, but $S$ becomes a biased, in particular transient, random walk with the following transition probabilities: For every basis vector $e \in \mathbf{Z}^{d}$,

$$
\mathrm{P}_{\lambda}\left\{S_{k+1}-S_{k}=e\right\}=\frac{\exp (\lambda \cdot e)}{\mathrm{E}\left(\exp \left(\lambda \cdot S_{1}\right)\right)} .
$$

As we did before, in the unforced setting, we consider the measures

$$
\mathrm{P}_{N}^{\beta, \lambda}(A):=\frac{1}{Z_{N}(\beta, \lambda)} \mathrm{E}_{\lambda}\left[\mathbf{1}_{A} \exp \left(\frac{\beta}{N} H_{N}\right) \mid q_{0}, q_{1}, \ldots, q_{N-1}\right],
$$


where $Z_{N}(\beta, \lambda)$ is the partition function,

$$
Z_{N}(\beta, \lambda):=\mathrm{E}_{\lambda}\left[\exp \left(\frac{\beta}{N} H_{N}\right) \mid q_{0}, q_{1}, \ldots, q_{N-1}\right]
$$

Then we proceed to define the " $\lambda$-analogues" of the quantities of interest. Namely:

$$
\begin{aligned}
\mathscr{D}_{\lambda} & :=\left\{\beta \in \mathbf{R}: Z_{N}(\beta, \lambda) \stackrel{\mathrm{P}}{\longrightarrow} \mathrm{e}^{\beta} \text { as } N \rightarrow \infty\right\} ; \\
\beta_{c}(\lambda) & :=\sup \mathscr{D}_{\lambda} ; \quad \text { and } \\
\digamma_{\lambda}(\beta) & :=\lim _{N \rightarrow \infty} \frac{1}{N} \ln Z_{N}(\beta, \lambda) .
\end{aligned}
$$

Of course, we can write $\mathrm{P}_{N}^{\beta, \lambda}(A)$ as follows as well:

$$
\mathrm{P}_{N}^{\beta, \lambda}(A)=\frac{\mathrm{E}\left[\mathbf{1}_{A} \exp \left(\frac{\beta}{N} H_{N}+\lambda \cdot S_{N-1}\right) \mid q_{0}, q_{1}, \ldots, q_{N-1}\right]}{Z_{N}(\beta, \lambda) \mathrm{E}\left(\exp \left(\lambda \cdot S_{1}\right)\right)^{N-1}} .
$$

The quantity $\lambda \cdot S_{N-1}$ is responsible for the different behavior of $\mathrm{P}_{N}^{\beta, \lambda}$ from $\mathrm{P}_{N}^{\beta}$, and corresponds to the potential energy of a pulling force $\lambda$.

Define

$$
I_{\lambda}(\varepsilon):=\lim _{N \rightarrow \infty} \frac{1}{N} \ln \mathrm{P}_{\lambda}\left\{L_{N}^{0}>\varepsilon N\right\} \quad \text { for all } \varepsilon \in(0,1 / 2) .
$$

The proof of Lemma A.1 below goes through, as no essential changes are necessary, and ensures that $I_{\lambda}:(0,1 / 2) \rightarrow(0, \infty)$ exists and is continuous.

We will see that Theorem 1.1, Proposition 1.4, and Theorem 1.5 continue to remain valid if we respectively replace $\mathscr{D}, \mathrm{P}, \mathrm{P}_{N}^{\beta}, \beta_{c}, \digamma$, and $I$ by $\mathscr{D}_{\lambda}$, $\mathrm{P}_{\lambda}, \mathrm{P}_{N}^{\beta, \lambda}, \beta_{c}(\lambda), \digamma_{\lambda}$, and $I_{\lambda}$.

We shall also prove that Theorems 1.7 and 1.8 continue to hold, but some of the stated constants need to be changed because the probability of the trajectory with maximal energy $H_{N}$ is no longer $(2 d)^{-N}$.

Our next result shows that the pulling force can sometimes trigger the folding/unfolding transition as $\beta_{c}(\lambda) \rightarrow \infty$ when $\lambda \rightarrow \infty$. It also prove 
that the function $\lambda \mapsto \beta_{c}(\lambda)$ is locally Lipschitz continuous. In order to prepare for that result let us observe that the right derivative $\digamma_{\lambda}^{\prime}$ of $\digamma_{\lambda}$ exists everywhere on $(0, \infty)$; this holds by convexity.

thm:pulling Theorem 1.13. If $\mathrm{E} q_{0}=0$, Var $q_{0}=1$, and $\kappa<\infty$, then:

1. For all $\lambda \in \mathbf{R}^{d}$,

$$
\begin{aligned}
& \beta_{c}(\lambda) \geqslant \kappa^{-1 / 2} \cdot\left[\sqrt{\frac{\ln \mathrm{E} \exp \left(\lambda \cdot S_{1}\right)}{\left(\mathrm{E} q_{0}^{+}\right)^{2}+\left(\mathrm{E} q_{0}^{-}\right)^{2}}} \vee \kappa^{-1 / 2}\right], \\
& \beta_{c}(\lambda) \leqslant \frac{2 \ln (2 d)\left(1+1_{\{1\}}(d)\right)+4\|\lambda\|_{\infty}}{\left(\mathrm{E} q_{0}^{+}\right)^{2}+\left(\mathrm{E} q_{0}^{-}\right)^{2}} .
\end{aligned}
$$

2. For all $\lambda, \mu \in \mathbf{R}^{d}$,

$$
\beta_{c}(\lambda+\mu)-\beta_{c}(\lambda) \leqslant \frac{2\|\mu\|_{\infty}}{\digamma_{\lambda}^{\prime}\left(\beta_{c}(\lambda)\right)}
$$

and $\digamma_{\lambda}^{\prime}\left(\beta_{c}(\lambda)\right)$ satisfies

$$
\digamma_{\lambda}^{\prime}\left(\beta_{c}(\lambda)\right) \geqslant \frac{1}{\beta_{c}(\lambda)} I_{\lambda}\left(\frac{1}{2 \kappa \beta_{c}(\lambda)}\right)
$$

\section{Proofs}

\subsection{Estimates on the partition function}

For every $\varepsilon>0$, we can consider the truncated partition function

$$
Z_{N}^{\varepsilon}(\beta):=\mathrm{E}\left[\mathbf{1}_{\left\{L_{N}^{\star} \leqslant \varepsilon N\right\}} \exp \left(\frac{\beta}{N} H_{N}\right) \mid q_{0}, q_{1}, \ldots, q_{N-1}\right] .
$$

The following is the main result of this subsection, and is essential to our characterization of the delocalized phase.

prop:EZ Proposition 2.1. Assume $\mathrm{E} q_{0}=0$ and $\operatorname{Var} q_{0}=1$. If $\varepsilon>0$ and $\beta \in \mathbf{R}$ satisfy either $\beta \leqslant 0$ or $2 \kappa \beta \varepsilon<1$, then $\lim _{N \rightarrow \infty} \operatorname{E} Z_{N}^{\varepsilon}(\beta)=\exp (\beta)$. 
Note that the above statement implies the convergence $\lim _{N \rightarrow \infty} \mathrm{E} Z_{N}(\beta)=$ $\exp (\beta)$ for any $\beta \in \mathbf{R}$ such that $\kappa \beta<1$, since $L_{N}^{\star} \leqslant(N+1) / 2$, and therefore $Z_{N}(\beta)=Z_{N}^{(1 / 2)+\delta}(\beta)$ for all $N \geqslant(2 \delta)^{-1}$.

The proof rests on two preparatory lemmas.

lem:Z:Jensen Lemma 2.2. Suppose $\mathrm{E} q_{0}=0$ and $\operatorname{Var} q_{0}=1$. Let $\varepsilon \in(0,1]$ and $\beta \in \mathbf{R}$ such that either $\beta \leqslant 0$ or $2 \kappa \beta \varepsilon<1$. Then, for all $\delta>0$, sufficiently small, there exists $C \in(0, \infty)$ such that for every $N \geqslant 1$, sufficiently large,

$$
\mathrm{E} \exp \left(\frac{\beta}{N}\left(q_{0}+\cdots+q_{l-1}\right)^{2}\right) \leqslant \exp \left(\beta \frac{l}{N}+\delta|\beta| \frac{l}{N}+C \frac{l^{2}}{N^{2}}\right),
$$

uniformly over $l \in\{1, \ldots,\lfloor\varepsilon N\rfloor\}$,

lem:moments Lemma 2.3. Choose and fix $\theta>0$. Then, as $N \rightarrow \infty$,

$$
\mathrm{E}\left[\exp \left(\frac{\theta}{N^{2}} \sum_{x \in \mathbf{Z}^{d}}\left(L_{N}^{x}\right)^{2}\right)\right] \leqslant 1+\delta_{N}
$$

where $\delta_{N}=O(\ln N / \sqrt{N})$ if $d=1, \delta_{N}=O\left([\ln N]^{2} / N\right)$ if $d=2$, and $\delta_{N}=O(\ln N / N)$ if $d \geqslant 3$.

Before we prove the two lemmas, let us use them in order to establish Proposition 2.1. The lemmas will be proved subsequently.

Proof of Proposition 2.1. Let us first note that for all possible realizations of $S:=\left\{S_{i}\right\}_{i=0}^{\infty}$,

$$
\mathrm{E}\left(H_{N} \mid S\right)=\sum_{x \in \mathbf{Z}^{d}} \mathrm{E}\left[\left(q_{1}+\cdots+q_{L_{N}^{x}}\right)^{2} \mid S\right]=\sum_{x \in \mathbf{Z}^{d}} L_{N}^{x}=N
$$

Therefore, Jensen's inequality implies that $\mathrm{E}\left[\exp \left(\beta H_{N} / N\right) \mid S\right] \geq \mathrm{e}^{\beta}$ for all realizations of $S$, whence

$$
\mathrm{E} Z_{N}^{\varepsilon}(\beta) \geqslant \mathrm{e}^{\beta} \mathrm{P}\left\{L_{N}^{\star} \leqslant \varepsilon N\right\} \rightarrow \mathrm{e}^{\beta} \quad \text { as } N \rightarrow \infty
$$

see Lemma A.2 below. This proves half of the assertion of the proposition. Next we establish a corresponding upper bound, thereby complete the proof. 
Thanks to Lemma 2.2, for all sufficiently small $\delta>0$ there exists a $C \in(0, \infty)$ such that for every $N \geqslant 1$, sufficiently large,

$$
\begin{aligned}
\mathrm{E} Z_{N}^{\varepsilon}(\beta) & =\mathrm{E}\left(\prod_{x \in \mathbf{Z}^{d}} \mathrm{E}\left[\exp \left(\frac{\beta}{N}\left(Q_{N}^{x}\right)^{2}\right) \mid S\right] \mathbf{1}_{\left\{L_{N}^{\star} \leqslant \varepsilon N\right\}}\right) \\
& \leqslant \mathrm{E}\left[\exp \left(\beta \sum_{x \in \mathbf{Z}^{d}} \frac{L_{N}^{x}}{N}+\delta|\beta| \sum_{x \in \mathbf{Z}^{d}} \frac{L_{N}^{x}}{N}+C \sum_{x \in \mathbf{Z}^{d}} \frac{\left(L_{N}^{x}\right)^{2}}{N^{2}}\right)\right] .
\end{aligned}
$$

Because $\sum_{x \in \mathbf{Z}^{d}} L_{N}^{x}=N$, it follows that

$$
\mathrm{E} Z_{N}^{\varepsilon}(\beta) \leqslant \mathrm{e}^{\beta+\delta|\beta|} \mathrm{E} \exp \left(C \sum_{x \in \mathbf{Z}^{d}} \frac{\left(L_{N}^{x}\right)^{2}}{N^{2}}\right)
$$

and the remainder of the proof follows then from Lemma 2.3.

Next, we set out to derive Lemmas 2.2 and 2.3, as promised earlier.

Proof of Lemma 2.2. Our goal is to derive a uniform estimate for

$$
\mathscr{E}:=\operatorname{E} \exp \left(\frac{\beta}{N}\left(q_{0}+\cdots+q_{l-1}\right)^{2}\right)
$$

[This is temporary notation, used specifically for this proof.]

Depending on the sign of $\beta$ we introduce the Laplace/Fourier transform

$$
\Psi(t):= \begin{cases}\operatorname{E} \exp \left(t q_{0}\right) & \text { if } \beta>0 \\ \operatorname{E} \exp \left(i t q_{0}\right) & \text { otherwise }\end{cases}
$$

The behavior of $\Psi$ at the origin is given by

$$
\Psi(t)=\exp \left(\operatorname{sgn}(\beta) \frac{t^{2}}{2}+o\left(t^{2}\right)\right) \quad \text { as } \quad t \rightarrow 0 .
$$


Furthermore, for all $t \in \mathbf{R}$,

$$
|\Psi(t)| \leqslant \begin{cases}\mathrm{e}^{\kappa t^{2} / 2} & \text { if } \beta>0 \\ 1 & \text { otherwise }\end{cases}
$$

Let $\xi$ be independent of $\left\{q_{i}\right\}_{i=0}^{\infty}$, and have a standard normal distribution. Then,

$$
\begin{aligned}
\mathscr{E}=\mathrm{E} \exp \left(\sqrt{\frac{2 \beta}{N}}\left(q_{0}+\cdots+q_{l-1}\right) \xi\right) & =\mathrm{E}\left(\Psi\left(\sqrt{\frac{2|\beta|}{N}} \xi\right)^{l}\right) \\
& \leqslant \mathrm{E}\left(\left|\Psi\left(\sqrt{\frac{2|\beta|}{N} \xi}\right)\right|^{l}\right) .
\end{aligned}
$$

According to (2.10), there exists some $A(\delta)>0$ such that

$$
|\Psi(t)| \leqslant \exp \left((\operatorname{sgn}(\beta)+\delta) \frac{t^{2}}{2}\right) \quad \text { when }|t| \leqslant A(\delta) .
$$

Because $\operatorname{E} \exp \left(a \xi^{2}\right)=(1-2 a)^{-1 / 2}$ for every $a<1 / 2,(2.12)$ implies that $\mathscr{E}$ is bounded above by

$$
\left[1-2(\beta+\delta|\beta|) \frac{l}{N}\right]^{-1 / 2}+\mathrm{E}\left[\exp \left(\varepsilon \kappa \beta^{+} \xi^{2}\right) \mathbf{1}_{\{|\xi|>A(\delta) \sqrt{N /(2 \beta)}\}}\right] .
$$

A Taylor expansion of the logarithm shows that if $\alpha<1 / 2$ then there exists $C \in(0, \infty)$ such that $-\frac{1}{2} \ln (1-2 \alpha x) \leqslant \alpha x+C x^{2}$ for all $x \in[0,1]$. Consequently if $\delta>0$ is sufficiently small, then $\ln \mathscr{E}$ is bounded above by

$$
\begin{aligned}
& (\beta+\delta|\beta|) \frac{l}{N}+C \frac{l^{2}}{N^{2}} \\
& \quad+\ln \left(1+\sqrt{1-2(\beta+\delta|\beta|) \frac{l}{N}} \mathrm{E}\left[\exp \left(\varepsilon \kappa \beta^{+} \xi^{2}\right) \mathbf{1}_{\{|\xi|>A(\delta) \sqrt{N /(2 \beta)}\}}\right]\right)
\end{aligned}
$$


and the logarithm is at most

$$
\sqrt{1+2|\beta|} \mathrm{E}\left[\exp \left(\varepsilon \kappa \beta^{+} \xi^{2}\right) \mathbf{1}_{\{|\xi|>A(\delta) \sqrt{N /(2 \beta)}}\right] .
$$

By the Cauchy-Schwarz inequality, the latter expectation vanishes exponentially fast as $N \rightarrow \infty$, because $\varepsilon \kappa \beta^{+}<1 / 2$; in particular, it is uniformly smaller than $l^{2} / N^{2}$ for all sufficiently large values of $N$. The lemma follows.

Proof of Lemma 2.3. Because $\sum_{x \in \mathbf{Z}^{d}}\left(L_{N}^{x}\right)^{2} \leqslant N L_{N}^{\star}$, it remains to bound $\mathrm{E}\left[\exp \left(\theta L_{N}^{\star} / N\right)\right]$. First of all, we note that for all $k \geqslant 0$ and $N \geqslant 1$,

$$
\begin{aligned}
\mathrm{E}\left[\left(L_{N}^{0}\right)^{k}\right] & =\sum_{0 \leqslant i_{1}, \ldots, i_{k}<N} \cdots \sum_{0 \leqslant i_{1} \leqslant \ldots \leqslant i_{k}<N} \mathrm{P}\left\{S_{i_{1}}=\cdots=S_{i_{k}}=0\right\} \\
& \left.\leqslant k ! \sum_{0} \cdots S_{i_{1}}=\cdots=S_{i_{k}}=0\right\} \\
& \leqslant k !\left(\mathrm{E} L_{N}^{0}\right)^{k} .
\end{aligned}
$$

Consequently,

$$
\mathrm{E}\left[\exp \left(\frac{L_{N}^{0}}{2 \mathrm{E} L_{N}^{0}}\right)\right]=\sum_{k=0}^{\infty} \frac{1}{k !} \mathrm{E}\left[\left(\frac{L_{N}^{0}}{2 \mathrm{E} L_{N}^{0}}\right)^{k}\right] \leqslant 2 .
$$

Therefore, Chebyshev's inequality, (2.17), and (A.8) together imply that for all $N \geqslant 1$ and $y>0$,

$$
\mathrm{P}\left\{L_{N}^{\star} \geqslant y N\right\} \leqslant 2(2 N)^{d} \exp \left(-\frac{y N}{2 \mathrm{E} L_{N}^{0}}\right) .
$$

We will use this bound only if the right-hand side is $\leqslant 1$; i.e., when

$$
y \geqslant \alpha_{N}, \quad \text { where } \quad \alpha_{N}:=\frac{2 \mathrm{E} L_{N}^{0} \times \ln \left[2(2 N)^{d}\right]}{N} .
$$


Else, we use the trivial bound $\mathrm{P}\left\{L_{N}^{\star} \geqslant y N\right\} \leqslant 1$. In this way, we find that

$$
\begin{aligned}
\int_{0}^{\infty} \mathrm{P}\left\{L_{N}^{\star} \geqslant y N\right\} \mathrm{e}^{\theta y} \mathrm{~d} y & \\
& \leqslant \alpha_{N}+O\left(N^{d}\right) \times \int_{\alpha_{N}}^{\infty} \exp \left\{\theta y-\frac{y N}{2 \mathrm{E} L_{N}^{0}}\right\} \mathrm{d} y .
\end{aligned}
$$

Since $\mathrm{E} L_{N}^{0}=\sum_{i=1}^{N} \mathrm{P}\left\{S_{i}=0\right\}$, the local-limit theorem [and excursion theory, when $d \geqslant 3$ ] together show that

$$
\mathrm{E} L_{N}^{0}=(1+o(1)) \times \begin{cases}\sqrt{N / \pi} & \text { if } d=1, \\ (2 \pi)^{-1} \ln N & \text { if } d=2, \\ 1 / \rho(d) & \text { if } d \geqslant 3\end{cases}
$$

where $\rho(d):=\mathrm{P}\left\{\inf _{k \geqslant 1}\left\|S_{k}\right\|>0\right\} \in(0,1)$ for $d \geqslant 3$. It follows readily from this and (2.20) that

$$
\alpha_{N}=(1+o(1)) \times \begin{cases}2 \ln N / \sqrt{\pi N} & \text { if } d=1, \\ 2(\ln N)^{2} /(\pi N) & \text { if } d=2, \\ 2 d \ln N /(\rho(d) N) & \text { if } d \geqslant 3 .\end{cases}
$$

Moreover,

$$
\begin{aligned}
\int_{0}^{\infty} \mathrm{P}\left\{L_{N}^{\star} \geqslant y N\right\} \mathrm{e}^{\theta y} \mathrm{~d} y & \\
& \leqslant \alpha_{N}+O\left(N^{d}\right) \times \int_{\alpha_{N}}^{\infty} \exp \left\{\theta y-\frac{y N}{2 \mathrm{E} L_{N}^{0}}\right\} \mathrm{d} y
\end{aligned}
$$

and direct computations show that the preceding is $O(\ln N / \sqrt{N})$ if $d=1$, $O\left([\ln N]^{2} / N\right)$ if $d=2$, and $O(\ln N / N)$ if $d \geqslant 3$. Integration by parts then shows that

$$
\mathrm{E}\left[\exp \left(\frac{\theta L_{N}^{\star}}{N}\right)\right]=1+\int_{0}^{\infty} \mathrm{P}\left\{L_{N}^{\star} \geqslant y N\right\} \mathrm{e}^{\theta y} \mathrm{~d} y
$$

Therefore, the lemma follows from the bound $\sum_{x \in \mathbf{Z}^{d}}\left(L_{N}^{x}\right)^{2} \leqslant N L_{N}^{\star}$. 


\subsection{The delocalized phase}

Before we give the proof of Theorem 1.1, we state and prove an easy consequence of Proposition 2.1:

lem:Z:eps Lemma 2.4. Assume $\mathrm{E} q_{0}=0$, Var $q_{0}=1$ and $\kappa<\infty$. Let $\varepsilon>0$ and $\beta \in \mathbf{R}$ such that either $\beta \leqslant 0$ or $2 \kappa \beta \varepsilon<1$. Then

$$
Z_{N}^{\varepsilon}(\beta) \stackrel{\mathrm{P}}{\longrightarrow} \mathrm{e}^{\beta} \quad \text { as } N \rightarrow \infty .
$$

Proof. First, we prove that, when $\beta \leqslant 0$ or $4 \kappa \beta \varepsilon<1$,

$$
Z_{N}^{\varepsilon}(\beta) \stackrel{L^{2}(\mathrm{P})}{\longrightarrow} \mathrm{e}^{\beta} \quad \text { as } N \rightarrow \infty
$$

Because $\left(Z_{N}^{\varepsilon}(\beta)\right)^{2} \leqslant Z_{N}^{\varepsilon}(2 \beta)$ [Jensen's inequality],

$$
\mathrm{E}\left(\left|Z_{N}^{\varepsilon}(\beta)-\mathrm{e}^{\beta}\right|^{2}\right) \leqslant \mathrm{E} Z_{N}^{\varepsilon}(2 \beta)+\mathrm{e}^{2 \beta}-2 \mathrm{e}^{\beta} \mathrm{E} Z_{N}^{\varepsilon}(\beta)
$$

The latter quantity goes to 0 as $N \rightarrow \infty$, thanks to Proposition 2.1, and this proves (2.26). Now we conclude the proof of the Lemma and assume $\beta \leqslant 0$ or $2 \kappa \beta \varepsilon<1$. The variable $Z_{N}^{\varepsilon}(\beta)-Z_{N}^{\varepsilon / 2}(\beta)$ is non-negative and its expectation goes to 0 as $N \rightarrow \infty$, cf. Proposition 2.1. Therefore it converges to 0 in probability. By $(2.26)$ we know already that $Z_{N}^{\varepsilon / 2}(\beta) \rightarrow \mathrm{e}^{\beta}$ in probability as $N \rightarrow \infty$. The conclusion follows.

Proof of Theorem 1.1. Let us first prove that $(-\infty, 1 / \kappa) \subseteq \mathscr{D}$. We choose and fix $\beta \in(-\infty, 1 / \kappa)$. There is $\delta>0$ such that $2 \kappa \beta\left(\frac{1}{2}+\delta\right)<1$. We have seen already that $Z_{N}(\beta)=Z_{N}^{(1 / 2)+\delta}(\beta)$ for all $N \geqslant(2 \delta)^{-1}$, therefore $\beta \in \mathscr{D}$ is a consequence of Lemma 2.4.

Next we prove that $\mathscr{D}$ is an interval. Thanks to the topology of $\mathbf{R}$, it suffices to show that $\mathscr{D} \cap(0, \infty)$ is connected.

Let us choose and fix $\beta_{1}, \beta_{2} \in \mathscr{D}$ such that $0<\beta_{1}<\beta_{2}$. For all $\beta \in$ $\left(\beta_{1}, \beta_{2}\right)$ and $\gamma>1,\left(Z_{N}(\beta)\right)^{\gamma} \leqslant Z_{N}(\gamma \beta)$, thanks to the conditional Jensen inequality. It follows that $Z_{N}\left(\beta_{1}\right)^{\beta / \beta_{1}} \leqslant Z_{N}(\beta) \leqslant Z_{N}\left(\beta_{2}\right)^{\beta_{2} / \beta}$. We can pass to the limit $[N \rightarrow \infty]$ to deduce that $\beta \in \mathscr{D}$. This implies the connectivity 
of $\mathscr{D}$, and completes the proof of part 1 .

Assertion 2 of the theorem holds because

$$
\mathrm{P}_{N}^{\beta}\left\{L_{N}^{\star} \leqslant \varepsilon N\right\}=\frac{Z_{N}^{\varepsilon}(\beta)}{Z_{N}(\beta)}
$$

and $Z_{N}^{\varepsilon}(\beta) \rightarrow \mathrm{e}^{\beta}$ in probability for all sufficiently small $\varepsilon>0$ [Lemma 2.4].

Finally we demonstrate part 3. Assume first $\beta \notin \mathscr{D}$. For $N$ fixed, the total variation is at least $\mathrm{P}_{N}^{\beta}\left\{L_{N}^{\star} \leqslant \varepsilon N\right\}-\mathrm{P}\left\{L_{N}^{\star} \leqslant \varepsilon N\right\}$, which does not converge to 0 in probability as $N \rightarrow \infty$ according to assertion 2 and to Lemma A.2.

Now we consider $\beta \in \mathscr{D}$ and $\varepsilon>0$ such that $4 \kappa \beta^{+} \varepsilon<1$, and consider some event $A$ that might depend on all $\left\{S_{i}\right\}_{i=0}^{\infty}$ and $\left\{q_{i}\right\}_{i=0}^{\infty}$. We have

$$
\left|\mathrm{P}_{N}^{\beta}(A)-\mathrm{P}\left(A \mid q_{0}, \ldots, q_{N-1}\right)\right| \leqslant d_{1}+d_{2}
$$

where

$$
\begin{aligned}
& d_{1}:=\left|\mathrm{P}_{N}^{\beta}\left(A \cap\left\{L_{N}^{\star} \leqslant \varepsilon N\right\}\right)-\mathrm{P}\left(A \cap\left\{L_{N}^{\star} \leqslant \varepsilon N\right\} \mid q_{0}, \ldots, q_{N-1}\right)\right|, \text { and } \\
& d_{2}:=\mathrm{P}_{N}^{\beta}\left(\left\{L_{N}^{\star}>\varepsilon N\right\}\right)+\mathrm{P}\left(\left\{L_{N}^{\star}>\varepsilon N\right\} \mid q_{0}, \ldots, q_{N-1}\right) .
\end{aligned}
$$

According to assertion 2 and to Lemma A.2, $d_{2} \rightarrow 0$ in probability as $N \rightarrow$ $\infty$. So it suffices to prove that $d_{1} \rightarrow 0$ in probability as $N \rightarrow \infty$, uniformly in $A$. It follows from the definition of $\mathrm{P}_{N}^{\beta}$ that

$$
\begin{aligned}
d_{1} & \leqslant \mathrm{E}\left[\left|\frac{\exp \left(\beta H_{N} / N\right)}{Z_{N}(\beta)}-1\right| \mathbf{1}_{A \cap\left\{L_{N}^{\star} \leqslant \varepsilon N\right\}} \mid q_{0}, q_{1}, \ldots, q_{N-1}\right] \\
& \leqslant \mathrm{E}\left[\left|\frac{\exp \left(\beta H_{N} / N\right)}{Z_{N}(\beta)}-1\right|^{2} \mathbf{1}_{\left\{L_{N}^{\star} \leqslant \varepsilon N\right\}} \mid q_{0}, q_{1}, \ldots, q_{N-1}\right]^{1 / 2} \\
& =\left[\frac{Z_{N}^{\varepsilon}(2 \beta)}{Z_{N}(\beta)^{2}}-2 \frac{Z_{N}^{\varepsilon}(\beta)}{Z_{N}(\beta)}+\mathrm{P}\left(\left\{L_{N}^{\star} \leqslant \varepsilon N\right\} \mid q_{0}, \ldots, q_{N-1}\right)\right]^{1 / 2} .
\end{aligned}
$$

And the latter quantity, which does not depend on $A$, goes to zero in probability as $N \rightarrow \infty$; see Lemma 2.4 and Lemma A.2. 
Finally we prove the invariance principle of the introduction.

Proof of Corollary 1.2. Theorem 1.1 implies that $\mathrm{E}_{N}^{\beta}\left[\Phi\left(\mathscr{S}_{N}\right)\right]-\mathrm{E}\left[\Phi\left(\mathscr{S}_{N}\right)\right]$ converges in probability to zero, as $N \rightarrow \infty$. And, according to Donsker's invariance principle, $\mathrm{E}\left[\Phi\left(\mathscr{S}_{N}\right)\right] \rightarrow \mathrm{E}[\Phi(\mathscr{B})]$. The corollary follows immediately from these observations.

\subsection{The existence of free energy (proof of Proposition 1.4)}

In this section we show that the normalized energy $H_{N} / N$ is subadditive, and then conclude Proposition 1.4 from that fact.

Lemma 2.5. Let $N_{1}, N_{2} \geqslant 1$ and $\tilde{q}:=\left\{q_{N+i}\right\}_{i=0}^{\infty}, \tilde{S}:=\left\{S_{N_{1}+i}-S_{N_{1}}\right\}_{i=0}^{\infty}$, $\tilde{Q}_{N}^{x}:=\sum_{i=0}^{N-1} \tilde{q}_{i} \mathbf{1}_{\left\{\tilde{S}_{i}=x\right\}}$, and $\tilde{H}_{N}:=\sum_{x \in \mathbf{Z}^{d}}\left(\tilde{Q}_{N}^{x}\right)^{2}$. Then,

$$
\frac{H_{N_{1}+N_{2}}}{N_{1}+N_{2}} \leqslant \frac{H_{N_{1}}}{N_{1}}+\frac{\tilde{H}_{N_{2}}}{N_{2}} \quad \text { a.s. }[\mathrm{P}] .
$$

Furthermore, $H_{N_{1}}$ and $H_{N_{2}}$ are conditionally independent, given $\left\{q_{i}\right\}_{i=0}^{\infty}$, and the conditional distribution of $\tilde{H}_{N_{2}}$ is the same as the conditional distribution of $\mathrm{H}_{\mathrm{N}_{2}}$ given the charges $\tilde{q}$.

Proof. Clearly,

$$
Q_{N_{1}+N_{2}}^{x}=Q_{N_{1}}^{x}+\tilde{Q}_{N_{2}}^{x+S_{N_{1}}} \quad \text { for every } x \in \mathbf{Z}^{d} .
$$

Therefore, the convexity of $h(x):=x^{2}$ implies that

$$
\frac{1}{N_{1}+N_{2}}\left(Q_{N_{1}+N_{2}}^{x}\right)^{2} \leqslant \frac{1}{N_{1}}\left(Q_{N_{1}}^{x}\right)^{2}+\frac{1}{N_{2}}\left(\tilde{Q}_{N_{2}}^{x+S_{N_{1}}}\right)^{2}
$$

We can sum the preceding over all $x \in \mathbf{Z}^{d}$ to deduce (2.32). In addition, the conditional distribution of $\tilde{H}_{N_{2}}$, given the charges $\tilde{q}$, depends only on the distribution of $\tilde{S}$, which is the law of a simple random walk.

Proof of Proposition 1.4. Let

$$
\digamma_{N}^{q}(\beta):=\frac{1}{N} \ln Z_{N}(\beta):=\frac{1}{N} \ln \mathrm{E}\left[\exp \left(\beta \frac{H_{N}}{N}\right) \mid q_{0}, q_{1}, \ldots, q_{N-1}\right]
$$


denote the free energy corresponding to a finite and fixed $N \geqslant 1$ and to a given realization of the charges $q:=\left\{q_{i}\right\}_{i=0}^{\infty}$.

By the conditional Jensen's inequality,

$$
\liminf _{N \rightarrow \infty} \mathrm{E}\left[\digamma_{N}^{q}(\beta)\right] \geqslant \beta \lim _{N} \mathrm{E}\left(\frac{H_{N}}{N^{2}}\right)=0
$$

since as $N \rightarrow \infty$,

$$
\mathrm{E} H_{N}=N \operatorname{Var}\left(q_{0}\right)+\left(\mathrm{E} q_{0}\right)^{2} \mathrm{E} \sum_{x \in \mathbf{Z}^{d}}\left(L_{N}^{x}\right)^{2}=o\left(N^{2}\right)
$$

see Lemma 2.3. This proves that if $\digamma(\beta)$ exists [as the proposition asserts] and is nonrandom, then certainly $\digamma(\beta) \geqslant 0$.

Now we prove convergence.

According to Lemma 2.5, for every fixed $N_{1}, N_{2} \geqslant 1$, we can bound $\digamma_{N_{1}+N_{2}}^{q}(\beta)$ from above by

$$
\begin{gathered}
\frac{1}{N_{1}+N_{2}} \ln \mathrm{E}\left[\exp \left(\beta \frac{H_{N_{1}}}{N_{1}}\right) \times \exp \left(\beta \frac{\tilde{H}_{N_{2}}}{N_{2}}\right) \mid q_{0}, q_{1}, \ldots, q_{N_{1}+N_{2}-1}\right] \\
=\frac{1}{N_{1}+N_{2}}\left(N_{1} \digamma_{N_{1}}^{q}(\beta)+N_{2} \digamma_{N_{2}}^{\tilde{q}}(\beta)\right) .
\end{gathered}
$$

Because $\digamma_{1}^{q}(\beta)=q_{0}^{2}$ has a finite expectation and because of the minoration (2.36), Kingman's subadditive ergodic theorem $[17,18]$ tells us that $\digamma_{N}^{q}(\beta)$ converges a.s. and in $L^{1}(\mathrm{P})$. In particular,

$$
\digamma(\beta)=\lim _{N \rightarrow \infty} \frac{1}{N} \mathrm{E} \ln Z_{N}(\beta) .
$$

The monotonicity and the convexity of $\beta \mapsto N^{-1} \ln Z_{N}(\beta)$, and hence of $\digamma$, follow respectively from the following relations:

$$
\begin{aligned}
\frac{\mathrm{d}}{\mathrm{d} \beta}\left(\digamma_{N}^{q}(\beta)\right) & =\frac{Z_{N}^{\prime}(\beta)}{N Z_{N}(\beta)}=\mathrm{E}_{N}^{\beta}\left(\frac{H_{N}}{N^{2}}\right) \\
\frac{\mathrm{d}^{2}}{\mathrm{~d} \beta^{2}}\left(\digamma_{N}^{q}(\beta)\right) & =\frac{Z_{N}^{\prime \prime}(\beta) Z_{N}(\beta)-\left[Z_{N}^{\prime}(\beta)\right]^{2}}{N\left[Z_{N}(\beta)\right]^{2}}=\operatorname{Var}_{\mathrm{P}_{N}^{\beta}}\left(\frac{H_{N}}{N^{3 / 2}}\right)
\end{aligned}
$$


together with the fact that both of these quantities are nonnegative.

\subsection{The first-order phase transition (proof of Theorem 1.5)}

Our proof of Theorem 1.5 requires three preliminary Lemmas.

lem: jump Lemma 2.6. For all $\beta>0$ and $\varepsilon, \eta>0$,

$$
\mathrm{P}_{N}^{\beta}\left\{\varepsilon<\frac{L_{N}^{\star}}{N} \leqslant \frac{1-\eta}{2 \kappa \beta}\right\} \stackrel{\mathrm{P}}{\longrightarrow} 0 \quad \text { as } N \rightarrow \infty
$$

Proof. We assume of course that $\varepsilon<(1-\eta) /(2 \kappa \beta)$. Because $Z_{N}(\beta) \geqslant 1$,

$$
\begin{aligned}
\mathrm{E}\left[\mathrm{P}_{N}^{\beta}\left\{\varepsilon<\frac{L_{N}^{\star}}{N} \leqslant \frac{1-\eta}{2 \kappa \beta}\right\}\right] & =\mathrm{E}\left[\frac{Z_{N}^{(1-\eta) /(2 \kappa \beta)}(\beta)-Z_{N}^{\varepsilon}(\beta)}{Z_{N}(\beta)}\right] \\
& \leqslant \mathrm{E}\left[Z_{N}^{(1-\eta) /(2 \kappa \beta)}(\beta)-Z_{N}^{\varepsilon}(\beta)\right] .
\end{aligned}
$$

This proves the lemma because according to Proposition 2.1 the preceding converges to zero as $N \rightarrow \infty$.

lem:densF Lemma 2.7. If $\mathrm{E}\left(q_{0}^{2}\right)=1$, then for all $\varepsilon, \beta>0$,

$$
\mathrm{P}_{N}^{\beta}\left\{\frac{L_{N}^{\star}}{N} \geqslant \frac{\digamma(\beta)}{\beta}-\varepsilon\right\} \stackrel{\mathrm{P}}{\longrightarrow} 1 \quad \text { as } N \rightarrow \infty .
$$

Proof. Whenever we have $H_{N} / N^{2} \leqslant-\varepsilon+[\digamma(\beta) / \beta]$, then we certainly have $\exp \left(\beta H_{N} / N\right) \leq \exp (N \digamma(\beta)-\beta \varepsilon N)$. Therefore,

$$
\mathrm{P}_{N}^{\beta}\left\{\frac{H_{N}}{N^{2}} \leqslant \frac{\digamma(\beta)}{\beta}-\varepsilon\right\} \leqslant \frac{\mathrm{e}^{N \digamma(\beta)-\beta \varepsilon N}}{Z_{N}(\beta)} .
$$

It follows from Proposition 1.4 that for every $\varepsilon>0$,

$$
\mathrm{P}_{N}^{\beta}\left\{\frac{H_{N}}{N^{2}} \geqslant \frac{\digamma(\beta)}{\beta}-\varepsilon\right\} \stackrel{\mathrm{P}}{\longrightarrow} 1 \quad \text { as } N \rightarrow \infty .
$$

Next we prove that the preceding implies the result. 
In accord with the Cauchy-Schwarz inequality,

$$
\begin{aligned}
\left(Q_{N}^{x}\right)^{2} & \leqslant\left(\sum_{i=1}^{N} q_{i}^{2} \mathbf{1}_{\left\{S_{i}=x\right\}}\right) \times L_{N}^{x} \quad \text { for all } x \in \mathbf{Z}^{d} \\
& \leqslant\left(\sum_{i=1}^{N} q_{i}^{2} \mathbf{1}_{\left\{S_{i}=x\right\}}\right) \times L_{N}^{\star} .
\end{aligned}
$$

We sum this inequality over $x \in \mathbf{Z}^{d}$ to find that

$$
H_{N} \leqslant L_{N}^{\star} \cdot \sum_{i=1}^{N} q_{i}^{2} .
$$

The lemma follows from (2.45) and the law of large numbers.

lem:LH Lemma 2.8. For every $\varepsilon, \beta>0$ and $0<\delta<I(\varepsilon) / \beta$,

$$
\mathrm{P}_{N}^{\beta}\left\{H_{N} \leqslant \delta N^{2}, L_{N}^{\star} \geqslant \varepsilon N\right\} \stackrel{\mathrm{P}}{\longrightarrow} 0 \quad \text { as } N \rightarrow \infty .
$$

Proof. According to Lemma A.2,

$$
\begin{aligned}
\limsup _{N \rightarrow \infty} \frac{1}{N} \ln \mathrm{E}\left[\mathrm{e}^{\beta H_{N} / N} \mathbf{1}_{\left\{H_{N} \leqslant \delta N^{2}, L_{N}^{\star} \geqslant \varepsilon N\right\}} \mid\right. & \left.q_{0}, \ldots, q_{N-1}\right] \\
& \leqslant \beta \delta-I(\varepsilon)<0,
\end{aligned}
$$

almost surely. Because $Z_{N}(\beta) \geqslant 1, \mathrm{P}_{N}^{\beta}\left\{H_{N} \leqslant \delta N^{2}, L_{N}^{\star} \geqslant \varepsilon N\right\}$ is a.s. bounded above by the conditional expectation in the preceding display.

Proof of Theorem 1.5. For all $\beta \in \mathbf{R}$ we define

$$
\gamma(\beta):=\lim _{\varepsilon \downarrow 0} \limsup _{N \rightarrow \infty} \mathrm{E}\left[\mathrm{P}_{N}^{\beta}\left\{\frac{L_{N}^{\star}}{N} \geqslant \varepsilon\right\}\right]
$$

Theorem 1.1 shows that $\gamma(\beta)>0$ if and only if $\beta \notin \mathscr{D}$. We will prove that, for all $\beta>0$,

$$
\lim _{\delta \downarrow 0} \frac{\digamma(\beta+\delta)-\digamma(\beta)}{\delta} \geqslant \frac{\gamma(\beta)}{\beta} I\left(\frac{1}{2 \kappa \beta}\right) .
$$

Before we address the proof of (2.51), we explain how it implies (1.18). For 
any $\beta>\beta_{c}$, we have $\gamma(\beta)>0$ [Theorem 1.1] and therefore a consequence of $(2.51)$ is that $\digamma(\beta)>0$, for all $\beta>\beta_{c}$. Then, from Lemma 2.7 it follows that $\digamma(\beta)>0 \Rightarrow \gamma(\beta)=1$, therefore $\gamma(\beta)=1$ for all $\beta>\beta_{c}$, and reporting in (2.51) yields the positive slope of $\digamma$ at the critical point, that is (1.18). Eq. (1.19) follows from the fact that $\digamma(\beta)>0$ for all $\beta>\beta_{c}$, together with Lemmas 2.7 and 2.6.

Now we turn to the proof of (2.51). We fix $\beta>0$ and $\varepsilon>0$. According to Lemma 2.6 we have as well

$$
\limsup _{N \rightarrow \infty} \mathrm{E}\left[\mathrm{P}_{N}^{\beta}\left\{\frac{L_{N}^{\star}}{N} \geqslant \frac{1-\varepsilon}{2 \kappa \beta}\right\}\right]=\gamma(\beta)
$$

Since $Z_{N}(\beta)$ and $Z_{N}^{\varepsilon}(\beta)$ are nondecreasing functions of $\beta$,

$$
\begin{aligned}
\inf _{\eta \in[0, \delta]} \mathrm{P}_{N}^{\beta+\eta}\left\{\frac{L_{N}^{\star}}{N}>\frac{1-\varepsilon}{2 \kappa \beta}\right\} \\
\geqslant 1-\frac{Z_{N}^{(1-\varepsilon) /(2 \kappa \beta)}(\beta+\delta)}{Z_{N}(\beta)} \\
\geqslant \mathrm{P}_{N}^{\beta}\left\{\frac{L_{N}^{\star}}{N}>\frac{1-\varepsilon}{2 \kappa \beta}\right\}-\varepsilon,
\end{aligned}
$$

almost surely on $\mathcal{T}_{N}^{\delta}$ where

$$
\mathcal{T}_{N}^{\delta}:=\left\{\frac{Z_{N}^{(1-\varepsilon) /(2 \kappa \beta)}(\beta+\delta)-Z_{N}^{(1-\varepsilon) /(2 \kappa \beta)}(\beta)}{Z_{N}(\beta)} \leqslant \varepsilon\right\} .
$$

According to Lemma 2.4, for all $\delta>0$ small enough, $Z_{N}^{(1-\varepsilon) /(2 \kappa \beta)}(\beta) \rightarrow \mathrm{e}^{\beta}$ while $Z_{N}^{(1-\varepsilon) /(2 \kappa \beta)}(\beta+\delta) \rightarrow \mathrm{e}^{\beta+\delta}$ in probability, as $N \rightarrow \infty$. Consequently $\mathrm{P}\left(\mathcal{T}_{N}^{\delta}\right) \rightarrow 1$ and

$$
\limsup _{N \rightarrow \infty} \inf _{\eta \in[0, \delta]} \mathrm{E}\left[\mathrm{P}_{N}^{\beta+\eta}\left\{\frac{L_{N}^{\star}}{N} \geqslant \frac{1-\varepsilon}{2 \kappa \beta}\right\}\right] \geqslant \gamma(\beta)-\varepsilon
$$

for all $\delta>0$ sufficiently small. In view of Lemma 2.8 , this yields also

$$
\limsup _{N \rightarrow \infty} \inf _{\eta \in[0, \delta]} \mathrm{E}\left[\mathrm{P}_{N}^{\beta+\eta}\left\{\frac{H_{N}}{N^{2}} \geqslant \frac{1}{\beta} I\left(\frac{1-\varepsilon}{2 \kappa \beta}\right)\right\}\right] \geqslant \gamma(\beta)-\varepsilon
$$


Consequently, we can integrate (2.40) over all $\eta \in(\beta, \beta+\delta)$ to see that

$$
\limsup _{N \rightarrow \infty}\left[\mathrm{E}\left[\digamma_{N}(\beta+\delta)\right]-\mathrm{E}\left[\digamma_{N}(\beta)\right]\right] \geqslant \delta \frac{\gamma(\beta)-\varepsilon}{\beta} I\left(\frac{1-\varepsilon}{2 \kappa \beta}\right)
$$

and letting $\varepsilon \rightarrow 0$ we conclude the proof of (2.51).

\subsection{Energy and the distance to optimality: The four points (Proofs of Proposition 1.6 and Theorem 1.7)}

The aim of this subsection is to prove Proposition 1.6 and Theorem 1.7. We consider henceforth the following related problem: What is the maximum value of $H_{N}$ given $q_{0}, \ldots, q_{N-1}$, where the maximum is taken over all possible random walk paths.

Let us introduce some notation. We say that $x \in \mathbf{Z}^{d}$ is odd (resp. even) when the sum of its coordinates is odd (resp. even). Given $N \geqslant 1$, $\varepsilon \in\{-,+\}$, and $p \in\{$ odd, even $\}$ we define

$$
Q_{\varepsilon}^{p}:=\sum_{\substack{0 \leqslant i<N: \\ i \equiv p}} q_{i}^{\varepsilon}
$$

where " $i \equiv p$ " means that " $i$ has parity $p . "$ The quantity $Q_{\varepsilon}^{p}$ is the total value of charges of $\operatorname{sign} \varepsilon$ available at positions of parity $p$.

Given a realization of $(q, S)$ we define $x_{\varepsilon}^{p}$ as any one of the points of $\mathbf{Z}^{d}$ with parity $p$ such that $\varepsilon Q_{N}^{x}$ is maximal (since positions with no charge exist we always have $\varepsilon Q_{N}^{x_{\varepsilon}^{p}} \geqslant 0$ ). It is not hard to see that we can ensure that $x_{\varepsilon}^{p}$ is always a random variable [measurable with respect to the sigma-algebra generated by $(q, S)]$.

Let us also observe that if there exists a point $x$ of parity $p$ such that $\varepsilon Q_{N}^{x}>Q_{\varepsilon}^{p} / 2$, then there is a unique choice for $x_{\varepsilon}^{p}$, namely $x_{\varepsilon}^{p}=x$.

We may think of

$$
D_{N}:=\sum_{\substack{\varepsilon \in\{-,+\} \\ p \in\{\text { odd,even }\}}} Q_{\varepsilon}^{p}\left(Q_{\varepsilon}^{p}-\varepsilon Q_{N}^{x_{\varepsilon}^{p}}\right)
$$


as the charge distance to optimality. Clearly, $D_{N} \geqslant 0$.

lem:HD Lemma 2.9. The following are valid for all $N \geqslant 1$ :

1. For every $d \geqslant 1$,

$$
H_{N} \leqslant \sum_{\substack{\varepsilon \in\{-,+\} \\ p \in\{\text { odd,even }\}}}\left(Q_{\varepsilon}^{p}\right)^{2}-D_{N}
$$

2. For every $d \geqslant 2$,

$$
\max _{S} H_{N}(S)=\sum_{\substack{\varepsilon \in\{-,+\} \\ p \in\{\text { odd,even }\}}}\left(Q_{\varepsilon}^{p}\right)^{2}
$$

where " $\max _{S}$ " refers to the maximum over all possible random walk paths.

Proof. In order to prove part 1 we first decompose, and then estimate, the energy as follows:

$$
\begin{aligned}
H_{N} & =\sum_{\substack{\varepsilon \in\{-,+\} \\
p \in\{\text { odd,even }\}}} \sum_{\substack{x \in \mathbf{Z}^{d}: x \equiv p, \varepsilon Q_{N}^{x}>0}}\left(Q_{N}^{x}\right)^{2} \\
& \leqslant \sum_{\substack{\varepsilon \in\{-,+\} \\
p \in\{\text { odd,even }\}}} \sum_{\substack{x \in \mathbf{Z}^{d}: x \equiv p, \varepsilon Q_{N}^{x}>0}}\left(\varepsilon Q_{N}^{x}\right) \times \sum_{\substack{x \in \mathbf{Z}^{d}: x \equiv p, \varepsilon Q_{N}^{x}>0}} \varepsilon Q_{N}^{x} \\
& \leqslant \sum_{\substack{\varepsilon \in\{-,+\} \\
p \in\{\text { odd,even }\}}} \varepsilon Q_{N}^{x_{\varepsilon}^{p}} \times Q_{\varepsilon}^{p} .
\end{aligned}
$$

We express the latter in terms of $D_{N}$ to complete the proof of part 1.

Next we demonstrate part 2.

Thanks to part 1 of the lemma,

$$
\max _{S} H_{N} \leqslant \sum_{\substack{\varepsilon \in\{-,+\} \\ p \in\{\text { odd,even }\}}}\left(Q_{\varepsilon}^{p}\right)^{2} \quad \text { for all } d \geqslant 1
$$


Now we assume $d \geqslant 2$ and describe an "optimal trajectory" in order to establish the second part of the lemma.

In order to be concrete, we will consider the case that $q_{0} \geqslant 0$; the case that $q_{0}<0$ can be considered similarly. Define

$$
\begin{aligned}
\sigma_{+}^{\text {even }}:=(0,0,0, \ldots), & \sigma_{-}^{\text {even }}:=(1,1,0, \ldots), \\
\sigma_{+}^{\text {odd }}:=(0,1,0, \ldots), & \sigma_{-}^{\text {odd }}:=(1,0,0, \ldots) .
\end{aligned}
$$

[When $q_{0}<0$, we exchange the roles of $\sigma_{+}^{\text {even }}$ and $\sigma_{-}^{\text {even }}$ in the following argument.] Now let us consider the following possible random walk trajectory:

$$
S_{i}=\sigma_{\operatorname{sgn}\left(q_{i}\right)}^{\operatorname{parity}(i)} \quad \text { for } i \geqslant 0
$$

A direct inspection shows that: (i) $S$ is a realization of the simple random walk; and (ii) this realization of the random walk path achieves the maximum energy $\max _{S} H_{N}$. [In particular, for this realization of the random walk we have $x_{\varepsilon}^{p}=\sigma_{\varepsilon}^{p}$.]

Our next Proposition is a ready consequence.

prop: $M$ Proposition 2.10. If $d \geqslant 2$, then a.s. $[\mathrm{P}]$,

$$
\lim _{N \rightarrow \infty} \max _{S} \frac{H_{N}}{N^{2}}=\frac{\left(\mathrm{E} q_{0}^{+}\right)^{2}+\left(\mathrm{E} q_{0}^{-}\right)^{2}}{2} \in[0, \infty]
$$

This result immediately implies Proposition 1.6 because the random walk piece $\left\{S_{i}\right\}_{i=0}^{N-1}$ is equal to the argmax of $S \mapsto H_{N}$ with probability $(2 d)^{-N}$. And therefore

$$
Z_{N}(\beta) \geqslant(2 d)^{-N} \exp \left(\beta \max _{S} \frac{H_{N}}{N}\right) .
$$

Proof of Proposition 2.10. Owing to Lemma 2.9, we can decompose the maximum energy as

$$
\max _{S} H_{N}=\left(Q_{+}^{\text {odd }}\right)^{2}+\left(Q_{-}^{\text {odd }}\right)^{2}+\left(Q_{+}^{\text {even }}\right)^{2}+\left(Q_{-}^{\text {even }}\right)^{2} .
$$

And one can check readily that the strong law of large numbers for i.i.d. 
nonnegative random variables implies that a.s. $[\mathrm{P}]$,

$$
\lim _{N \rightarrow \infty} \frac{Q_{\varepsilon}^{p}}{N / 2}=\mathrm{E} q_{0}^{\varepsilon} \quad \text { for all } \varepsilon \in\{-,+\} \text { and } p \in\{\text { odd, even }\}
$$

This completes the proof.

Next we present a lower bound for $D_{N}$ in terms of four nonadjacent points. This bound will play an important role in the proof of Theorem 1.7. It also will lead to an upper bound on the maximum energy $\max _{S} H_{N}$ in the case that $d=1$.

lem-Dxd1 Lemma 2.11. If $d \geqslant 1$ and $\varepsilon, \varepsilon^{\prime} \in\{-,+\}$ satisfy $\left\|x_{\varepsilon}^{\text {odd }}-x_{\varepsilon^{\prime}}^{\text {even }}\right\| \neq 1$, then

$$
D_{N} \geqslant \sum_{1 \leqslant i<N: i \text { odd }} \min \left(Q_{\varepsilon}^{\text {odd }} q_{i}^{\varepsilon}, Q_{\varepsilon^{\prime}}^{\text {even }} q_{i-1}^{\varepsilon^{\prime}}\right) \text {. }
$$

Proof. First of all, let us observe from the definition of $D$ that

$$
D_{N} \geqslant Q_{\varepsilon}^{\text {odd }}\left(Q_{\varepsilon}^{\text {odd }}-\varepsilon Q_{N}^{x_{\varepsilon}^{\text {odd }}}\right)+Q_{\varepsilon^{\prime}}^{\text {even }}\left(Q_{\varepsilon^{\prime}}^{\text {even }}-\varepsilon^{\prime} Q_{N}^{x_{\varepsilon^{\prime}}^{\text {even }}}\right) .
$$

Next we note that

$$
\begin{gathered}
Q_{\varepsilon}^{\text {odd }}-\varepsilon Q_{N}^{x_{\varepsilon}^{\text {odd }}} \geqslant \sum_{i \text { odd }: S_{i} \neq x_{\varepsilon}^{\text {odd }}} q_{i}^{\varepsilon}, \quad \text { and } \\
Q_{\varepsilon^{\prime}}^{\text {even }}-\varepsilon^{\prime} Q_{N}^{x_{\varepsilon^{\prime}}^{\text {even }}} \geqslant \sum_{i \text { even: }: S_{i} \neq x_{\varepsilon^{\prime}}^{\text {even }}} q_{i}^{\varepsilon^{\prime}} .
\end{gathered}
$$

If $i \in\{1, \ldots, N-1\}$ is odd, then we necessarily have either $S_{i-1} \neq x_{\varepsilon^{\prime}}^{\text {even }}$ or $S_{i} \neq x_{\varepsilon}^{\text {odd }}$. Therefore, the lemma follows.

The following lemma will also be useful in our forthcoming analysis.

lem:IwbH Lemma 2.12. For all $d \geqslant 1, \beta \geqslant 0, \varepsilon>0, N \geqslant 1$, and $q_{0}, \ldots, q_{N-1} \in \mathbf{R}$ :

$$
\mathrm{P}_{N}^{\beta}\left\{\max _{S} H_{N}-H_{N} \geqslant \varepsilon N^{2}\right\} \leqslant \mathrm{e}^{N[\ln (2 d)-\beta \varepsilon]} .
$$


Proof. Because

$$
\mathrm{P}_{N}^{\beta}\left\{\max _{S} H_{N}-H_{N} \geqslant \varepsilon N^{2}\right\} \leqslant \frac{\exp \left(\frac{\beta}{N}\left(\max _{S} H_{N}-\varepsilon N^{2}\right)\right)}{Z_{N}(\beta)},
$$

the lemma follows from (2.70).

Now we conclude the proof of Theorem 1.7. We introduce

$$
\begin{aligned}
\Gamma & :=\min _{\substack{\varepsilon \in\{-,+\} \\
p \in\{\text { even,odd }\}}}\left(Q_{\varepsilon}^{p}\right)^{2}, \\
\Lambda & :=\min _{\varepsilon, \varepsilon^{\prime} \in\{-,+\}} \sum_{0 \leqslant i<N: i \text { odd }} \min \left(Q_{\varepsilon}^{\text {odd }} q_{i}^{\varepsilon}, Q_{\varepsilon^{\prime}}^{\text {even }} q_{i-1}^{\varepsilon^{\prime}}\right) .
\end{aligned}
$$

Recall from (1.22) and (1.23) the quantities $\gamma$ and $\lambda$. Then a direct inspection reveals that

$$
\lim _{N \rightarrow \infty} \frac{\Gamma}{(N / 2)^{2}}=\gamma, \quad \lim _{N \rightarrow \infty} \frac{\Lambda}{(N / 2)^{2}}=\lambda
$$

For every fixed $\delta>0$, let us consider the events

$$
\begin{aligned}
\mathcal{E}_{N}^{\delta} & :=\left\{(1+\delta) \frac{\Gamma}{N^{2}} \geqslant \frac{\gamma}{4} \text { and }(1+\delta) \frac{\Lambda}{N^{2}} \geqslant \frac{\lambda}{4}\right\}, \\
\mathcal{C}_{\alpha}: & =\left\{\begin{array}{l}
\varepsilon Q_{N}^{x_{\varepsilon}^{p}} \geqslant \frac{1+\alpha}{2} Q_{\varepsilon}^{p} \text { for all } \varepsilon= \pm \text { and } p=\text { odd/even } \\
\left\|x_{\varepsilon}^{\text {odd }}-x_{\varepsilon^{\prime}}^{\text {even }}\right\|=1 \text { for all } \varepsilon, \varepsilon^{\prime}= \pm 1
\end{array}\right\},
\end{aligned}
$$

so that $\mathcal{C}_{\alpha}$ is the event that the points $x_{ \pm}^{\text {odd / even }}$ are adjacent and possess each a proportion at least $(1+\alpha) / 2$ of the available charge. Note, in particular, that

$$
\mathcal{C}_{\alpha} \subseteq \mathcal{S}_{\alpha},
$$

where the event $\mathcal{S}_{\alpha}$ was defined in (2.82).

Proof of Theorem 1.7. In accord with Cramér's theorem there exists $c_{\delta}>0$ such that

$$
\mathrm{P}\left(\mathcal{E}_{N}^{\delta}\right) \geqslant 1-\exp \left(-c_{\delta} N\right) \quad \text { for all } N \geqslant 1 \text {. }
$$


Next we observe that if $\varepsilon Q_{N}^{x_{\varepsilon}^{p}} \leqslant(1+\alpha) Q_{\varepsilon}^{p} / 2$ for some $p \in\{$ odd, even $\}$ and $\varepsilon \in\{-,+\}$, then

$$
D_{N} \geqslant\left(\frac{1-\alpha}{2}\right)\left(Q_{\varepsilon}^{p}\right)^{2} \geqslant\left(\frac{1-\alpha}{2}\right) \Gamma
$$

in accord with the definition (2.59) of $D_{N}$. If, on the other hand, $\| x_{\varepsilon}^{\text {odd }}-$ $x_{\varepsilon^{\prime}}^{\text {even }} \| \neq 1$ for some $\varepsilon, \varepsilon^{\prime} \in\{-,+\}$ then $D_{N} \geqslant \Lambda$ [Lemma 2.11]. Therefore, we may apply Lemmas 2.9 and 2.12 in conjunction to deduce that the following holds almost surely on $\mathcal{E}_{N}^{\delta}$ :

$$
\begin{aligned}
\mathrm{P}_{N}^{\beta}\left(\mathcal{C}_{\alpha}^{c}\right) & \leqslant \mathrm{P}_{N}^{\beta}\left\{D_{N} \geqslant \min \left(\frac{1-\alpha}{2} \cdot \Gamma, \Lambda\right)\right\} \\
& \leqslant \mathrm{P}_{N}^{\beta}\left\{(1+\delta) \frac{D_{N}}{N^{2}} \geqslant \min \left(\frac{1-\alpha}{2} \cdot \frac{\gamma}{4}, \frac{\lambda}{4}\right)\right\} \\
& \leqslant \exp \left(N\left[\ln (2 d)-\frac{\beta}{1+\delta} \min \left(\frac{1-\alpha}{2} \cdot \frac{\gamma}{4}, \frac{\lambda}{4}\right)\right]\right) .
\end{aligned}
$$

This and (2.82) together imply the result.

Our next result estimates the maximum allowable energy $\max _{S} H_{N} / N^{2}$ in the case that $d=1$. It might help to recall that $\lambda$ was defined in (1.23).

lem:maxH:d1 Lemma 2.13. If $d=1$, then

$$
\limsup _{N \rightarrow \infty} \frac{1}{N^{2}} \max _{S} H_{N} \leqslant \frac{\left(\mathrm{E} q_{0}^{+}\right)^{2}+\left(\mathrm{E} q_{0}^{-}\right)^{2}}{2}-\frac{\lambda}{4} \quad \text { a.s. }[\mathrm{P}]
$$

And, for all $\varepsilon \in\{-,+\}$,

$$
\begin{aligned}
& \liminf _{N \rightarrow \infty} \frac{1}{N^{2}} \max _{S} H_{N} \\
& \geqslant \frac{\left(\mathrm{E} q_{0}^{+}\right)^{2}+\left(\mathrm{E} q_{0}^{-}\right)^{2}}{4}+\frac{a_{4}}{4}\left(\mathrm{E} q_{0}^{\varepsilon}\right)^{2}+\left(\frac{\mathrm{E} q_{0}}{2}-\frac{\varepsilon a_{2} \mathrm{E} q_{0}^{\varepsilon}}{2}\right)^{2}
\end{aligned}
$$

almost sure $[\mathrm{P}]$, where

$$
a_{k}:=\left[\mathrm{P}\left\{q_{0} \geqslant 0\right\}\right]^{k}+\left[\mathrm{P}\left\{q_{0}<0\right\}\right]^{k} \quad \text { for } k=2,4 .
$$


Remark 2.14. In the case that $q_{0}$ has the Rademacher distribution [i.e., $\left.\mathrm{P}\left\{q_{0}= \pm 1\right\}=1 / 2\right]$, the preceding tells us that

$$
\frac{19}{128} \leqslant \liminf _{N \rightarrow \infty} \frac{1}{N^{2}} \max _{S} H_{N} \leqslant \limsup _{N \rightarrow \infty} \frac{1}{N^{2}} \max _{S} H_{N} \leqslant \frac{7}{32} .
$$

[Note that $19 / 128 \approx 0.1484375$ and $7 / 32 \approx 0.21875$.]

Proof of Lemma 2.13. We use the same notation as in the former proof. Since we have $d=1$ it is not possible that $x_{ \pm}^{\text {odd }}$ are adjacent to $x_{ \pm}^{\text {even }}$. In view of Lemma 2.11 this implies that

$$
(1+\delta) \frac{D_{N}}{N^{2}} \geqslant \frac{\lambda}{4} \quad \text { for all } q \in \mathcal{E}_{N}^{\delta},
$$

and hence $\max _{S} H_{N} / N^{2}$ is bounded above by

$$
\frac{\left(Q_{+}^{\text {odd }}\right)^{2}+\left(Q_{-}^{\text {odd }}\right)^{2}+\left(Q_{+}^{\text {even }}\right)^{2}+\left(Q_{-}^{\text {even }}\right)^{2}}{N^{2}}-\frac{\lambda}{4(1+\delta)},
$$

for every $q \in \mathcal{E}_{N}^{\delta}$. This yields the first assertion of the lemma.

We propose the following strategy in order to establish the asserted [asymptotic] lower bound on $N^{-2} \max _{S} H_{N}$ : Choose and fix a sign $\varepsilon \in\{ \pm\}$, and place odd monomers at positions $S_{i}=1$ if $q_{i} \geqslant 0, S_{i}=-1$ otherwise, and even monomers - whenever possible [that is, $S_{i-1}=S_{i+1}$ ] at position $S_{i}= \pm 2$ if $\operatorname{sign}\left(q_{i}\right)=\varepsilon$ and $S_{i}=0$ otherwise. A computation, involving the strong law of large numbers, then shows that almost surely $[\mathrm{P}]$,

$$
\begin{aligned}
& \lim _{N \rightarrow \infty} \frac{Q_{N}^{ \pm 1}}{N}= \pm \frac{\mathrm{E} q_{0}^{ \pm}}{2}, \\
& \lim _{N \rightarrow \infty} \frac{Q_{N}^{+2}}{N}=\varepsilon \frac{\mathrm{E} q_{0}^{\varepsilon}}{2}\left(\mathrm{P}\left\{q_{0} \geqslant 0\right\}\right)^{2}, \\
& \lim _{N \rightarrow \infty} \frac{Q_{N}^{-2}}{N}=\varepsilon \frac{\mathrm{E} q_{0}^{\varepsilon}}{2}\left(\mathrm{P}\left\{q_{0}<0\right\}\right)^{2}, \quad \text { and } \\
& \lim _{N \rightarrow \infty} \frac{Q_{N}^{0}}{N}=\frac{\mathrm{E} q_{0}}{2}-\varepsilon a_{2} \frac{\mathrm{E} q_{0}^{\varepsilon}}{2} .
\end{aligned}
$$

This yields the lower bound. 


\subsection{Logarithmic range and bounded expectation of $\left|S_{N}\right|$ (Proofs of Theorems 1.8 and 1.11)}

In this Section we prove Theorem 2.15 below and derive Theorem 1.8 from it. We also present here a proof of Theorem 1.11.

Given $N \geqslant 1$ and $L \geqslant 1$, define

$$
\overline{\mathrm{q}}_{L}:=\min _{\substack{\ell \geqslant L \\ 0 \leqslant i<N-\ell}}\left(\frac{1}{\ell} \sum_{k=i}^{i+\ell-1}\left|q_{k}\right|\right) .
$$

Theorem 2.15 (Logarithmic diameter). 1. If $d \geqslant 1$ and $\mathrm{E}\left|q_{0}\right|<\infty$, then for every $\beta \in \mathbf{R}$ and $\varepsilon>0$ there exists $c>0$ such that for all sufficiently large integers $N \geqslant 1$,

$$
\mathrm{E}\left[\mathrm{P}_{N}^{\beta}\left\{\frac{\operatorname{Diam}\left\{S_{i}: 0 \leqslant i<N\right\}}{\ln N} \geqslant c\right\}\right] \geqslant 1-\exp \left(-c N^{1-\varepsilon}\right) .
$$

2. If $d \geqslant 2$, then for every $\alpha \in(0,1), N \geqslant 1,0 \leqslant L \leqslant N$, and $\beta \in \mathbf{R}$,

$$
\begin{aligned}
\mathrm{P}_{N}^{\beta}\left(\left\{\operatorname { D i a m } \left\{S_{i}: 0\right.\right.\right. & \left.\leqslant i<N\} \geqslant L+1\} \cap \mathcal{C}_{\alpha}\right) \\
& \leqslant N^{2} \exp \left(L\left[\ln (2 d)-\frac{2 \beta \alpha \sqrt{\Gamma}}{N} \overline{\mathrm{q}}_{L}\right]\right) .
\end{aligned}
$$

First we present a quick proof of Theorem 1.8 that uses Theorem 2.15. Then we establish the latter result.

Proof of Theorem 1.8. We apply (2.95) and (2.96) with $L:=C \ln N$ to obtain all but part 2 immediately. And part 2 follows from Theorem 1.7 and from Cramér's theorem, since

$$
\mathrm{P}\left\{\overline{\mathrm{q}}_{L} \leqslant \mathrm{E}\left|q_{0}\right|-\varepsilon\right\} \leqslant N^{2} \sup _{l \geqslant L} \mathrm{P}\left\{\frac{\left|q_{1}\right|+\cdots+\left|q_{l}\right|}{l} \leqslant \mathrm{E}\left|q_{0}\right|-\varepsilon\right\}
$$

decays more quickly than $N^{-K}$, provided that $C$ is large enough.

Our proof of Theorem 2.15 hinges on an analysis of the trajectory of a 
certain portion of the polymer, conditional on the charges and the remaining portions of the polymer. We begin with a Lemma that is useful for bounding the range of the polymer from above.

Choose and fix an integer $N \geqslant 1$, and let $I$ be a contiguous subset of $\{0, \ldots, N-1\}$ with $|I|<N$. Given a realization of the polymer $S$ that satisfies $C_{\alpha}$ for some $0<\alpha<1$, we say that monomer $i \in\{0, \ldots, N-1\}$ is optimal when $S_{i}=x_{\operatorname{sgn}\left(q_{i}\right)}^{\text {parity }(i)}$ (when $q_{i}=0$, monomer $i$ is optimal when $\left.S_{i} \in\left\{x_{+}^{\text {parity }(i)}, x_{-}^{\text {parity }(i)}\right\}\right)$. By extension, we say that $S$ is nonoptimal on $I$ when none of the monomers $i \in I$ are optimal. Define

$$
\begin{aligned}
\mathcal{N}(I) & :=\{S \text { is nonoptimal on } I\}, \text { and } \\
\mathcal{C}(I) & :=\mathcal{C}_{\alpha} \cap\{S \text { is optimal at the position(s) next to } I\}
\end{aligned}
$$

where $\alpha \in(0,1)$, and $\mathcal{C}_{\alpha}$ is the event defined in $(2.81)$.

lem:HSt Lemma 2.16. Let $N, \alpha$, and $I$ be fixed as above. Given a realization of $q$ and $S \in \mathcal{N}(I) \cap \mathcal{C}(I)$, define $\tilde{S}$ as follows:

$$
\tilde{S}_{i}= \begin{cases}S_{i} & \text { if } i \notin I \\ x_{+}^{\text {parity }(i)} & \text { if } i \in I \& q_{i} \geqslant 0 \\ x_{-}^{\text {parity }(i)} & \text { if } i \in I \& q_{i}<0\end{cases}
$$

Then the trajectory $\left(\tilde{S}_{i}-\tilde{S}_{0}\right)_{i=0}^{N-1}$ is a possible realization of a simple random walk and

$$
H_{N}(\tilde{S})-H_{N}(S) \geqslant 2 \alpha \sum_{\substack{\varepsilon \in\{-,+\} \\ p \in\{\text { odd,even }\}}}\left[Q_{\varepsilon}^{p} \times \sum_{i \in I: i \equiv p} q_{i}^{\varepsilon}\right]
$$

Proof. Because $S$ is optimal off $I, \tilde{S}$ is a simple random walk [but it might not start at the origin]. 
Next we decompose $H_{N}(\tilde{S})-H_{N}(S)$ as

$$
\begin{aligned}
& \sum_{x \in\left\{x_{ \pm}^{\text {odd even }}\right\} \cup\left\{S_{i} ; i \in I\right\}}\left[\left(Q_{N}^{x}(\tilde{S})\right)^{2}-\left(Q_{N}^{x}(S)\right)^{2}\right] \\
= & \sum_{x \in\left\{x_{ \pm}^{\text {odd } / \text { even }}\right\} \cup\left\{S_{i} ; i \in I\right\}}\left(Q_{N}^{x}(\tilde{S})+Q_{N}^{x}(S)\right)\left(Q_{N}^{x}(\tilde{S})-Q_{N}^{x}(S)\right) .
\end{aligned}
$$

Now we observe that: (i) If $x=x_{\varepsilon}^{p}$, then

$$
Q_{N}^{x}(\tilde{S})=Q_{N}^{x}(S)+\sum_{i \in I: i \equiv p, \varepsilon q_{i}>0} q_{i} ;
$$

and (ii) If $x=S_{i}$ for some $i \in I$, then

$$
Q_{N}^{x}(\tilde{S})=Q_{N}^{x}(S)-\sum_{i \in I: S_{i}=x} q_{i}
$$

Consequently, we can write

$$
H_{N}(\tilde{S})-H_{N}(S):=T_{1}-T_{2},
$$

where

$$
T_{1}:=\sum_{\substack{\varepsilon \in\{-,+\} \\ p \in\{\text { odd,even }\}}}\left(2 Q_{N}^{x_{\varepsilon}^{p}}(S)+\sum_{i \in I: i \equiv p, \varepsilon q_{i}>0} q_{i}\right) \sum_{i \in I: i \equiv p, \varepsilon q_{i}>0} q_{i}
$$

and

$$
T_{2}:=\sum_{x \in\left\{S_{i} ; i \in I\right\}}\left(Q_{N}^{x}(\tilde{S})+Q_{N}^{x}(S)\right) \sum_{i \in I: S_{i}=x} q_{i} .
$$

Since $\varepsilon Q_{N}^{x_{\varepsilon}^{p}}(S) \geqslant(1+\alpha) / 2 Q_{\varepsilon}^{p}(S)$,

$$
T_{1} \geqslant(1+\alpha) \sum_{\substack{\varepsilon \in\{-,+\} \\ p \in\{\text { odd,even }\}}} Q_{\varepsilon}^{p} \times \sum_{i \in I: i \equiv p} q_{i}^{\varepsilon} .
$$


Let us write, temporarily,

$$
\mathcal{X}^{\text {odd }}:=\left\{S_{i} ; i \in I \text { odd }\right\}
$$

Then clearly

$$
\begin{aligned}
& \sum_{x \in \mathcal{X}^{\text {odd }}}\left(Q_{N}^{x}(\tilde{S})+Q_{N}^{x}(S)\right) \sum_{i \in I: S_{i}=x} q_{i} \\
& \leqslant \sum_{\varepsilon \in\{-,+\}} \sum_{x \in \mathcal{X}_{\text {odd }}}\left(Q_{N}^{x}(\tilde{S})+Q_{N}^{x}(S)\right)^{\varepsilon}\left[\sum_{i \in I: S_{i}=x} q_{i}\right]^{\varepsilon} \\
& \leqslant \sum_{\varepsilon \in\{-,+\}} \max _{\text {odd: } x \neq x_{ \pm}^{\text {odd }}}\left(Q_{N}^{x}(\tilde{S})+Q_{N}^{x}(S)\right)^{\varepsilon} \times \sum_{i \in I: i \text { odd }} q_{i}^{\varepsilon} \\
& \leqslant(1-\alpha) \sum_{\varepsilon \in\{-,+\}} Q_{\varepsilon}^{\text {odd }} \sum_{i \in I: i \text { odd }} q_{i}^{\varepsilon}
\end{aligned}
$$

the last line is valid because, whenever $x \neq x_{ \pm}^{\text {odd }}$ is odd, the quantities $Q_{N}^{x}(\tilde{S})$ and $Q_{N}^{x}(S)$ both lie in the interval $\left[-\frac{1}{2}(1-\alpha) Q_{-}^{\text {odd }}, \frac{1}{2}(1-\alpha) Q_{+}^{\text {odd }}\right]$. It follows that

$$
T_{2} \leqslant(1-\alpha) \sum_{\substack{\varepsilon \in\{-,+\} \\ p \in\{\text { odd,even }\}}} Q_{\varepsilon}^{p} \sum_{i \in I: i \equiv p} q_{i}^{\varepsilon} .
$$

The claims follows from (2.104), (2.107), and (2.110).

Proof of Theorem 2.15. We begin by deriving (2.95).

With probability exponentially close to one [as $N \rightarrow \infty$ ], the total charge of the polymer satisfies

$$
\sum_{i=0}^{N-1}\left|q_{i}\right| \leqslant 2 N \mathrm{E}\left|q_{0}\right| .
$$

Therefore, by conditioning, we may [and will] assume that the charges satisfy the former inequality.

Because the $q$ 's satisfy (2.111), it follows that if we modify a single position $S_{i}$ of the polymer, then we change $H_{N}(S)$ by at most $8 N \mathrm{E}\left|q_{0}\right| \times\left|q_{i}\right|$. 
Consequently,

$$
\begin{array}{r}
\mathrm{P}_{N}^{\beta}\left(\begin{array}{c|c}
\operatorname{Diam}\left\{S_{i}: N_{1} \leqslant i<N_{2}\right\} & \left.S_{0}, \ldots S_{N_{1}-1}, S_{N_{2}}, \ldots, S_{N-1}\right) \\
\geqslant \frac{1}{(2 d)^{N_{2}-N_{1}}} \exp \left(-8|\beta| \mathrm{E}\left|q_{0}\right| \sum_{N_{1} \leqslant i<N_{2}}\left|q_{i}\right|\right)
\end{array},\right.
\end{array}
$$

almost surely for every $N_{1}<N_{2}$ in $\{0, \ldots, N\}$. Given $L \in\{1, \ldots, N-1\}$, define

$$
\mathcal{K}_{L}:=\left\{k \in\{1, \ldots,[N / L]\}: \sum_{(k-1) L \leqslant i<k L}\left|q_{i}\right| \leqslant 2 L \mathrm{E}\left|q_{0}\right|\right\} .
$$

Then, (2.112) leads to the bound

$$
\begin{aligned}
\mathrm{P}_{N}^{\beta}\left\{\max _{k \in \mathcal{K}_{L}} \operatorname{Diam}\left\{S_{i}:(k-1) L<i \leqslant k L\right\}<\frac{L-1}{2}\right\} & \\
& \leqslant\left(1-a^{L}\right)^{\left|\mathcal{K}_{L}\right|}
\end{aligned}
$$

where $a:=\exp \left(-16|\beta|\left(\mathrm{E}\left|q_{0}\right|\right)^{2}\right) /(2 d)$. Now we choose $L$ judiciously; namely, we let $L:=L_{N}:=[-\varepsilon \ln (N) / \ln (a)]$ - so that $a^{L} / N^{-\varepsilon} \rightarrow 1$ as $N \rightarrow \infty$-in order to deduce the following:

$$
\begin{aligned}
& \mathrm{E}\left[\mathrm{P}_{N}^{\beta}\left\{\operatorname{Diam}\left\{S_{i}: i<N\right\}<\frac{L-1}{2}\right\}\right] \\
& \leqslant \mathrm{P}\left\{\sum_{i=1}^{N}\left|q_{i}\right|>2 N \mathrm{E}\left|q_{0}\right|\right\}+\mathrm{P}\left\{\left|\mathcal{K}_{L}\right| \leqslant \frac{N}{2 L}\right\}+\left(1-N^{-\varepsilon}\right)^{N /(2 L)} .
\end{aligned}
$$

This yields (2.95).

We prove (2.96) next.

If $\mathcal{C}_{\alpha}$ holds and $S$ has $L$ consecutive nonoptimal monomers, then we can find a contiguous $I \subset\{0, \ldots, N-1\}$ such that $L \leqslant|I|<N$ and $S \in \mathcal{N}(I) \cap \mathcal{C}(I)$. There are not more than $N^{2}$ corresponding choices for 
such an $I$. Therefore,

$$
\begin{aligned}
& \mathrm{P}_{N}^{\beta}\left(\left\{\begin{array}{l}
S \text { has } L \text { consecutive } \\
\text { nonoptimal monomers }
\end{array}\right\} \cap \mathcal{C}_{\alpha}\right) \\
& \leqslant N^{2} \times \sup _{I \text { contiguous: }|I| \geqslant L} \mathrm{P}_{N}^{\beta}(\mathcal{N}(I) \cap \mathcal{C}(I)) .
\end{aligned}
$$

Consider such a contiguous set $I$. Every $S \in \mathcal{N}(I) \cap \mathcal{C}(I)$ gets mapped to $\bar{S}:=\tilde{S}-\tilde{S}_{0} \in \mathcal{C}(I)$, and no more than $(2 d)^{|I|}$ choices of $S$ yield the same $\bar{S}$. In addition, Lemma 2.16 and the definition (2.78) of $\Gamma$ together tell us that

$$
\begin{aligned}
H_{N}(\tilde{S})-H_{N}(S) & \geqslant 2 \alpha \sum_{\substack{\varepsilon \in\{-,+\} \\
p \in\{\text { odd,even }\}}}\left[\sqrt{\Gamma} \times \sum_{i \in I: i \equiv p} q_{i}^{\varepsilon}\right] \\
& =2 \alpha \sqrt{\Gamma} \sum_{i \in I}\left|q_{i}\right| .
\end{aligned}
$$

Therefore,

$$
\begin{aligned}
& \mathrm{P}_{N}^{\beta}(\mathcal{N}(I) \cap \mathcal{C}(I)) \\
& \leqslant \exp \left(-2 \beta \alpha \frac{\sqrt{\Gamma}}{N} \sum_{i \in I}\left|q_{i}\right|\right) \frac{1}{Z_{N}^{\beta}} \sum_{S \in \mathcal{N}(I) \cap \mathcal{C}(I)} \exp \left(-\beta H_{N}(\tilde{S}) / N\right) \\
& \leqslant \exp \left(-2 \beta \alpha \frac{\sqrt{\Gamma}}{N} \sum_{i \in I}\left|q_{i}\right|\right)(2 d)^{|I|} \mathrm{P}_{N}^{\beta}(\mathcal{C}(I)) \\
& \leqslant \exp \left(|I| \times\left[\ln (2 d)-2 \beta \alpha \frac{\sqrt{\Gamma}}{N} \overline{\mathrm{q}}_{L}\right]\right)
\end{aligned}
$$

owing to the definition of $\overline{\mathrm{q}}_{L}$. The claim follows from this and (2.116).

Proof of Theorem 1.11. The proof is similar to the proof of Theorem 2.15.

Recall that $R_{\alpha}^{N}$ was defined in (1.30), and define $I:=\{0, \ldots, r-1\}$ for some fixed $1 \leqslant r<N$. Then, we may use (2.118) and the obvious fact that 
$\mathrm{P}_{N}^{\beta}(\mathcal{C}(I)) \leqslant 1$ in order to deduce that

$$
\begin{aligned}
\mathrm{P}_{N}^{\beta}\left(\left\{R_{\alpha}^{N}=r\right\} \cap \mathcal{C}_{\alpha}\right) & =\mathrm{P}_{N}^{\beta}(\mathcal{N}(I) \cap \mathcal{C}(I)) \\
& \leqslant \exp \left(-2 \beta \alpha \frac{\sqrt{\Gamma}}{N} \sum_{i=0}^{r-1}\left|q_{i}\right|\right)(2 d)^{r} .
\end{aligned}
$$

Next, we choose and fix an arbitrary $\delta>0$., and recall from (2.80) the event $\mathcal{E}_{N}^{\delta}$. Then almost surely on $\mathcal{E}_{N}^{\delta}$,

$$
\mathrm{P}_{N}^{\beta}\left(\left\{R_{\alpha}^{N}=r\right\} \cap \mathcal{C}_{\alpha}\right) \leqslant(2 d)^{r} \exp \left(-2 \beta \alpha \sqrt{\frac{\gamma}{1+\delta}} \sum_{i=0}^{r-1}\left|q_{i}\right|\right)
$$

Because $R_{\alpha}^{N} \leqslant N$, it follows that

$$
\begin{aligned}
\mathrm{E}\left[\mathrm{E}_{N}^{\beta}\left(R_{\alpha}^{N}\right)\right] \leqslant N\left[1-\mathrm{P}\left(\mathcal{E}_{N}^{\delta}\right)\right]+N \mathrm{E}\left[1-\mathrm{P}_{N}^{\beta}\left(\mathcal{C}_{\alpha}\right)\right] \\
+\sum_{r=0}^{N-1} r \mathrm{E}\left[\mathbf{1}_{\mathcal{E}_{N}^{\delta}} \mathrm{P}_{N}^{\beta}\left(\left\{R_{\alpha}^{N}=r\right\} \cap \mathcal{C}_{\alpha}\right)\right] \\
\leqslant o(1)+\sum_{r=1}^{N-1} r(2 d)^{r} \mathrm{E}\left[\exp \left(-2 \beta \alpha \sqrt{\frac{\gamma}{1+\delta}} \sum_{i=0}^{r-1}\left|q_{i}\right|\right)\right]
\end{aligned}
$$

as $N \rightarrow \infty$; see (2.83) and (2.85). Define

$$
\rho_{\delta}:=2 d \mathrm{E}\left[\exp \left(-\beta \alpha \sqrt{\frac{\gamma}{1+\delta}}\left|q_{0}\right|\right)\right] .
$$

Since $\lim _{\delta \downarrow 0} \rho_{\delta}=\rho$, it follows that $\rho_{\delta}<1$ for all $\delta>0$ sufficiently small. And hence, for all $\delta>0$ sufficiently small,

$$
\limsup _{N \rightarrow \infty} \mathrm{E}\left[\mathrm{E}_{N}^{\beta}\left(R_{\alpha}^{N}\right)\right] \leqslant \sum_{r=1}^{\infty} r \rho_{\delta}^{r}=\frac{\rho_{\delta}}{\left(1-\rho_{\delta}\right)^{2}} .
$$

Let $\delta \rightarrow 0$ to finish. 


\subsection{On the annealed measure}

Our analysis of the quenched measure can be adapted with no difficulty, and with some simplifications, to study also the annealed measure. Here we prove only Proposition 1.12.

Proof of Proposition 1.12. We know already from the analog of Theorem 1.1 that $\tilde{\beta}_{c} \geqslant 1$. Therefore, it suffices to prove that $\mathrm{E} Z_{N}(1)=\infty$. Let

$$
\nu:=\nu(N):=\lceil N / 2\rceil
$$

Because $\mathrm{P}\left\{L_{N}^{\star}=\nu\right\} \geqslant(2 d)^{-N}$ for all $N$ sufficiently large, it follows immediately from properties of the normal distribution that

$$
\begin{aligned}
\mathrm{E} Z_{N}(1) & \geqslant(2 d)^{-N} \mathrm{E}\left[\exp \left\{\frac{\left(q_{1}+\cdots+q_{\nu}\right)^{2}}{N}\right\}\right] \\
& =(2 d)^{-N} \mathrm{Ee}^{\nu q_{0}^{2} / N} .
\end{aligned}
$$

And the latter quantity is infinite because $\nu / N \geqslant 1 / 2$.

\subsection{The influence of a pulling force}

First we justify our claim that the results of Theorem 1.1, Proposition 1.4, and Theorem 1.5 continue to hold [up to a modification of the notation]. Basically, this is so because Lemma 2.3 is the only place where we explicitly used the fact that $S$ is the simple symetric random walk. Now the new measure $\mathrm{P}_{\lambda}$ has the following property:

$$
\mathrm{P}_{\lambda}\left\{S_{k}=0\right\}=\frac{\mathrm{P}\left\{S_{k}=0\right\}}{\left(\mathrm{E} \exp \left(\lambda \cdot S_{1}\right)\right)^{k}},
$$

with $\operatorname{E} \exp \left(\lambda \cdot S_{1}\right)>1$ whenever $\lambda \neq 0$. Therefore, the local time at the origin satisfies $\mathrm{E}_{\lambda} L_{N}^{0} \leqslant \mathrm{E} L_{N}^{0}$. This is enough for concluding that even the statement of Lemma 2.3 continues to hold when we replace $\mathrm{E}$ with $\mathrm{E}_{\lambda}$ for $\lambda \in \mathbf{R}^{d}$. Next we prove Theorem 1.13.

Proof of Theorem 1.13. We begin with the proof of (1.45): We know already 
that $\beta_{c}(\lambda) \geqslant 1 / \kappa$. [Theorem 1.1]. Let us choose and fix some $\varepsilon>0$. Then we can write

$$
\begin{aligned}
& Z_{N}(\beta, \lambda) \\
& \quad=Z_{N}^{(1-\varepsilon) /(2 \kappa \beta)}(\beta, \lambda)+\mathrm{E}_{\lambda}\left[\mathrm{e}^{\beta H_{N} / N^{\prime}} \mathbf{1}_{\mathcal{A}(N)} \mid q_{0}, q_{1}, \ldots, q_{N-1}\right],
\end{aligned}
$$

where $Z_{N}^{\varepsilon}(\beta, \lambda)$ is defined by adapting (2.1) - in the obvious way - to the new reference measure $\mathrm{P}_{\lambda}$, and $\mathcal{A}(N)$ denotes the following event:

$$
\mathcal{A}(N):=\left\{\frac{L_{N}^{\star}}{N}>\frac{1-\varepsilon}{2 \kappa \beta}\right\} .
$$

We know from Theorem 1.1 that $Z_{N}^{(1-\varepsilon) /(2 \kappa \beta)}(\beta, \lambda) \rightarrow \mathrm{e}^{\beta}$ in probability as $N \rightarrow \infty$. Next we consider the second term in (2.128).

Because

$$
\begin{aligned}
\mathrm{E}_{\lambda}\left[\exp \left(\frac{\beta}{N} H_{N}\right) \mathbf{1}_{\mathcal{A}(N)} \mid q_{0}, q_{1}, \ldots, q_{N-1}\right] \\
\leqslant \exp \left(\beta N \max _{S} \frac{H_{N}}{N^{2}}\right) \mathrm{P}_{\lambda}\left\{\frac{L_{N}^{\star}}{N}>\frac{1-\varepsilon}{2 \kappa \beta}\right\},
\end{aligned}
$$

it follows that

$$
\digamma_{\lambda}(\beta) \leqslant \max \left(\beta \frac{\left(\mathrm{E} q_{0}^{+}\right)^{2}+\left(\mathrm{E} q_{0}^{-}\right)^{2}}{2}-I_{\lambda}\left(\frac{1-\varepsilon}{2 \kappa \beta}\right), 0\right)
$$

where $I_{\lambda}$ was defined in (1.44).

We conclude the proof by establishing a lower bound for $I_{\lambda}$.

According to (2.127),

$$
\mathrm{E}_{\lambda} L_{\infty}^{0} \leqslant \frac{1}{1-1 / \mathrm{E}\left(\exp \left(\lambda \cdot S_{1}\right)\right)}
$$

By the strong Markov property, $L_{\infty}^{0}$ has a geometric distribution with parameter

$$
p:=\mathrm{P}_{\lambda}\left\{S_{i} \neq 0 \text { for all } i \geqslant 1\right\}
$$


and therefore $p \geqslant 1-1 / \mathrm{E}\left(\exp \left(\lambda \cdot S_{1}\right)\right)$. It follows that

$$
\mathrm{P}_{\lambda}\left\{L_{N}^{0} \geqslant \alpha N\right\} \leqslant \mathrm{P}_{\lambda}\left\{L_{\infty}^{0} \geqslant \alpha N\right\}=(1-p)^{\lceil\alpha N\rceil-1},
$$

and consequently

$$
I_{\lambda}(\alpha) \geqslant \alpha \ln \mathrm{E}\left(\exp \left(\lambda \cdot S_{1}\right)\right)
$$

The conclusion (1.45) is immediate. Now we address the opposite bound (1.46).

If $e \in \mathbf{Z}^{d}$ has norm 1 , then

$$
\mathrm{P}_{\lambda}\left\{S_{k+1}-S_{k}=e\right\} \geqslant \frac{\exp \left(-2\|\lambda\|_{\infty}\right)}{2 d}
$$

whence

$$
Z_{N}(\beta, \lambda) \geqslant \exp \left(\beta N \max _{S} \frac{H_{N}}{N^{2}}\right) \times\left(\frac{\exp \left(-2\|\lambda\|_{\infty}\right)}{2 d}\right)^{N} .
$$

Consequently, (1.46) follows from Proposition 2.10 when $d \geqslant 2$; and (1.46) follows from Lemma 2.13 when $d=1$.

Finally, we prove that $\beta_{c}$ is locally Lipschitz.

The density

$$
\left.\frac{\mathrm{dP}_{\lambda+\mu}}{\mathrm{dP}_{\lambda}}\right|_{\sigma\left(q, S_{0}, \ldots, S_{k}\right)}:=\frac{\exp \left(\mu \cdot S_{k}\right)}{\left(\mathrm{E}_{\lambda} \exp \left(\mu \cdot S_{1}\right)\right)^{k}}
$$

is bounded from above and below respectively by $\exp \left( \pm 2 k\|\mu\|_{1}\right)$. Therefore, for all $\beta \in \mathbf{R}$,

$$
\begin{aligned}
Z_{N}(\beta, \lambda+\mu) & \geqslant Z_{N}(\beta, \lambda) \exp \left(-2 N\|\mu\|_{\infty}\right), \\
\digamma_{\lambda+\mu}(\beta) & \geqslant \digamma_{\lambda}(\beta)-2\|\mu\|_{\infty} .
\end{aligned}
$$

This proves the claim when one chooses

$$
\beta>\beta_{c}(\lambda)+2 \frac{\|\mu\|_{\infty}}{\digamma_{\lambda}^{\prime}\left(\beta_{c}(\lambda)\right)}
$$


for which $\digamma_{\lambda+\mu}(\beta)>0$ [thanks to the convexity of $\digamma_{\lambda+\mu}$ ]. The lower bound on $\digamma_{\lambda}^{\prime}\left(\beta_{c}(\lambda)\right)$ comes from the generalization of (1.19) in Theorem 1.5.

\section{A The local times of the random walk}

In this appendix we collect some facts about the local times of the simple random walk $\left\{S_{i}\right\}_{i=0}^{\infty}$ on $\mathbf{Z}^{d}$. Recall that the local time at $x$ of the walk is denoted by the process $\left\{L_{N}^{x}\right\}_{N=1}^{\infty}$, and is defined by $L_{N}^{x}:=\sum_{0 \leqslant i<N} \mathbf{1}_{\left\{S_{i}=x\right\}}$.

lem:LTO Lemma A.1. There exists a continuous nondecreasing function $I:(0,1 / 2) \rightarrow$ $(0, \infty)$ such that

$$
\lim _{N \rightarrow \infty} \frac{1}{N} \ln \mathrm{P}\left\{L_{N}^{0}>\varepsilon N\right\}=-I(\varepsilon) \quad \text { for all } \varepsilon \in(0,1 / 2) .
$$

In fact, the limit exists for all $\varepsilon>0$. But the additional gain in generality is uninteresting because $\mathrm{P}\left\{L_{N}^{0}>\varepsilon N\right\}=0$-whence $I(\varepsilon)=\infty$-when $\varepsilon \geqslant$ $1 / 2$, since the simple walk on $\mathbf{Z}^{d}$ has period 2 .

Proof. Let $\tau_{0}:=0$ and for $k \geqslant 1$ define $\tau_{k}$ to be the $k$ th return time to the origin by the random walk; that is, $\tau_{k}:=\min \left\{j>\tau_{k-1}: S_{j}=0\right\}$. It is easy to see that $L_{N}^{0}>\varepsilon N$ if and only if $\tau_{[\varepsilon N]}<N$. According to a result of Jain and Pruitt [14, Theorem 2.1],

$$
\lim _{N \rightarrow \infty} \frac{1}{N} \mathrm{P}\left\{\tau_{[\varepsilon N]}<N\right\}=-R\left(g^{-1}(1 / \varepsilon)\right) \quad \text { for all } \varepsilon \in(0,1 / 2),
$$

where $R$ is continuous, $g$ is continuous and strictly decreasing, and both are defined as follows:

$$
g(u):=-\frac{\varphi^{\prime}(u)}{\varphi(u)} \quad \text { and } \quad R(u):=-\ln \varphi(u)-u g(u),
$$

where $\varphi(u)=\mathrm{E} \exp \left(-u \tau_{1}\right)$. This implies our lemma with

$$
I(\varepsilon):=\left(R \circ g^{-1}\right)(1 / \varepsilon) \quad \text { for all } \varepsilon \in(0,1 / 2) .
$$

Let us also mention that $\varphi$ can be computed, in a standard way, by appealing 
to excursion theory [16, Lemma 2.1]. The end-result is that

$$
\varphi(u)=\frac{1}{(2 \pi)^{d}} \int_{(-\pi, \pi)^{d}} \frac{\mathrm{d} \xi}{1-G(\xi) \mathrm{e}^{-u}},
$$

where $G(\xi):=d^{-1} \sum_{j=1}^{d} \cos \left(\xi \cdot \mathbf{e}_{j}\right)$ for the $d$ standard basis vectors $\left\{\mathbf{e}_{j}\right\}_{j=1}^{d}$ of $\mathbf{R}^{d}$. We omit the details of this standard computation.

Recall that $L_{N}^{\star}:=\sup _{x \in \mathbf{Z}^{d}} L_{N}^{x}$ denotes the maximum local time.

lem:LTstar Lemma A.2. For every fixed $x \in \mathbf{Z}^{d}, L_{N}^{x}$ is stochastically smaller than $L_{N}^{0}$. Therefore, for the same function I as in Lemma A.1,

$$
\lim _{N \rightarrow \infty} \frac{1}{N} \mathrm{P}\left\{L_{N}^{\star}>\varepsilon N\right\}=-I(\varepsilon) \quad \text { for all } \varepsilon \in(0,1 / 2) .
$$

Proof. Recall that the assertion about stochastic monotonicity is simply that $\mathrm{P}\left\{L_{N}^{x}>a\right\} \leqslant \mathrm{P}\left\{L_{N}^{0}>a\right\}$ for all $a \in \mathbf{Z}^{d}$. This is a ready consequence of the strong Markov property [applied at the first hitting time of the origin]. Because

$$
\mathrm{P}\left\{L_{N}^{0}>a\right\} \leqslant \mathrm{P}\left\{L_{N}^{\star}>a\right\} \leqslant \sum_{\substack{x \in \mathbf{Z}^{d}: \\\|x\|_{1} \leqslant n}} \mathrm{P}\left\{L_{N}^{x}>a\right\},
$$

stochastic monotonicity implies that for all $N \geqslant 1$,

$$
\mathrm{P}\left\{L_{N}^{0}>a\right\} \leqslant \mathrm{P}\left\{L_{N}^{\star}>a\right\} \leqslant(2 N)^{d} \mathrm{P}\left\{L_{N}^{0}>a\right\}
$$

Therefore, Lemma A.1 finishes the proof.

Acknowledgements. We are grateful to Amine Asselah and Bernard Derrida for references and helpful discussions. It is a pleasure as well to thank Nicolas Petrelis for his helpful comments on the first-order phase transition, and Dmitry Ioffe for suggesting that we include the material in Section 1.6. We are also grateful to Romain Abraham for suggesting the present formulation of part 3 of Theorem 1.1. 


\section{References}

A08 [1] A. Asselah. Annealed upper tails for the energy of a polymer. arXiv:0812.0443, 2008.

A09 [2] A. Asselah. Annealed lower tails for the energy of a polymer. arXiv:0909.5291, 2009.

C08 [3] X. Chen. Limit laws for the energy of a charged polymer. Ann. Inst. Henri Poincaré Probab. Stat., 44(4):638-672, 2008.

CK09 [4] X. Chen and D. Khoshnevisan. From charged polymers to random walk in random scenary. IMS Lecture Notes. Optimality: The 3rd E.L. Lehmann Symposium, 57:237-251, 2009.

CSY04 [5] F. Comets, T. Shiga, and N. Yoshida. Probabilistic analysis of directed polymers in a random environment: a review. In Stochastic analysis on large scale interacting systems, volume 39 of Adv. Stud. Pure Math., pages 115-142. Math. Soc. Japan, Tokyo, 2004.

[6] F. den Hollander. Random polymers, volume 1974 of Lecture Notes in Mathematics. Springer-Verlag, Berlin, 2009. Lectures from the 37th Probability Summer School held in Saint-Flour, 2007.

[7] B. Derrida, R. B. Griffiths, and P. G. Higgs. A model of directed walks with random self-interactions. EPL (Europhysics Letters), 18(4):361, 1992.

DH94 [8] B. Derrida and P. G. Higgs. Low-temperature properties of directed walks with random self interactions. Journal of Physics A: Mathematical and General, 27(16):5485, 1994.

G088b [9] T. Garel and H. Orland. Chemical sequence and spatial structure in simple models of biopolymers. EPL (Europhysics Letters), 6(7):597-601, 1988.

G088a [10] T. Garel and H. Orland. Mean-field model for protein folding. EPL (Europhysics Letters), 6(4):307-310, 1988.

Gia07 [11] G. Giacomin. Random Polymer Models. Imperial College Press, London, 2007.

GK97 [12] I. Golding and Y. Kantor. Two-dimensional polymers with random short-range interactions. Phys. Rev. E, 56(2):R1318-R1321, Aug 1997.

HK10 [13] Yueyun Hu and Davar Khoshnevisan. Strong approximations in a chargedpolymer model. Periodica Mathematica Hungaria, 61(1-2):213-224, 2010. 
JP87 [14] Naresh C. Jain and William E. Pruitt. Lower tail probability estimates for subordinators and nondecreasing random walks. Ann. Probab., 15(1):75-101, 1987.

KK91 [15] Y. Kantor and M. Kardar. Polymers with random self-interactions. EPL (Europhysics Letters), 14(5):421, 1991.

[16] Davar Khoshnevisan. A discrete fractal in $\mathbf{Z}_{+}^{1}$. Proc. Amer. Math. Soc., 120(2):577-584, 1994.

K68 [17] J. F. C. Kingman. The ergodic theory of subadditive stochastic processes. J. Roy. Statist. Soc. Ser. B, 30:499-510, 1968.

K73 [18] J. F. C. Kingman. Subadditive ergodic theory. Ann. Probability, 1:883-909, 1973. With discussion by D. L. Burkholder, Daryl Daley, H. Kesten, P. Ney, Frank Spitzer and J. M. Hammersley, and a reply by the author.

[19] R. van der Hofstad and W. König. A survey of one-dimensional random polymers. J. Statist. Phys., 103(5-6):915-944, 2001.

Yueyun Hu. Département de Mathématiques, Université Paris XIII, 99 avenue JB Clément, F-93430 Villetaneuse, France, Email: yueyun@math.univ-paris13.fr

Davar Khoshnevisan. Department of Mathematics, University of Utah, 155 South 1440 East, JWB 233, Salt Lake City, Utah 84112-0090, USA,

Email: davar@math.utah.edu

Marc Wouts. Département de Mathématiques, Université Paris XIII, 99 avenue J-

B Clément, F-93430 Villetaneuse, France, Email: wouts@math.univ-paris13.fr 